\title{
A machine learning framework for TCP round-trip time estimation
}

\author{
Bruno Astuto Arouche Nunes ${ }^{1 *}$, Kerry Veenstra ${ }^{1}$, William Ballenthin ${ }^{2}$, Stephanie Lukin ${ }^{3}$ and Katia Obraczka
}

\begin{abstract}
In this paper, we explore a novel approach to end-to-end round-trip time (RTT) estimation using a machine-learning technique known as the experts framework. In our proposal, each of several 'experts' guesses a fixed value. The weighted average of these guesses estimates the RTT, with the weights updated after every RTT measurement based on the difference between the estimated and actual RTT.

Through extensive simulations, we show that the proposed machine-learning algorithm adapts very quickly to changes in the RTT. Our results show a considerable reduction in the number of retransmitted packets and an increase in goodput, especially in more heavily congested scenarios. We corroborate our results through 'live' experiments using an implementation of the proposed algorithm in the Linux kernel. These experiments confirm the higher RTT estimation accuracy of the machine learning approach which yields over $40 \%$ improvement when compared against both standard transmission control protocol (TCP) as well as the well known Eifel RTT estimator. To the best of our knowledge, our work is the first attempt to use on-line learning algorithms to predict network performance and, given the promising results reported here, creates the opportunity of applying on-line learning to estimate other important network variables.
\end{abstract}

\section{Introduction}

Latency is an important parameter when designing, managing, and evaluating computer networks, their protocols, and applications. One metric that is commonly used to capture network latency is the end-to-end round-trip time (RTT) which measures the time between data transmission and the receipt of a positive acknowledgment. Depending on how the RTT is measured (e.g., at which layer of the protocol stack), besides the time it takes for the data to be serviced by the network, the RTT also accounts for the 'service time' at the communication end points. In some cases, RTT measurement can be done implicitly by using existing messages; however, in several instances, explicit 'probe' messages have to be used. Such explicit measurement techniques can render the RTT estimation process quite expensive in terms of their communication and computational burden.

Several network applications and protocols use the RTT to estimate network load or congestion and therefore need

\footnotetext{
${ }^{*}$ Correspondence: bastuto@gmail.com

1 Department of Computer Engineering, Baskin School of Engineering,

University of California, Santa Cruz, CA 95064, USA

Full list of author information is available at the end of the article
}

to measure it frequently. The transmission control protocol, TCP, is one of the best known examples. TCP bases its error, flow, and congestion control functions on the estimated RTT instead of relying on feedback from the network. This pure end-to-end approach to network control is consistent with the original design philosophy of the Internet which keeps only the bare minimum functionality in the network core, pushing everything else to the edges. Overlays such as content distribution networks (CDNs) (e.g., Akamai [1]) and peer-to-peer networks also make use of RTT as a 'network proximity' metric, e.g., to help decide where to re-direct client requests. There has also been increasing interest in network proximity information from applications that run on mobile devices (e.g., smart phones) in order to improve the user's experience.

In this paper, we propose a novel RTT estimation technique that uses a machine-learning based approach called the experts framework [2]. As described in detail in Section 3, the experts framework ${ }^{\mathrm{a}}$ uses 'on-line' learning, where the learning process happens in trials. At every trial, a number of experts contribute to an overall prediction, which is compared to the actual value of the RTT (e.g., obtained by measurement). The algorithm uses the

\section{它 Springer}

(c) 2014 Nunes et al.: licensee Springer. This is an Open Access article distributed under the terms of the Creative Commons Attribution License (http://creativecommons.org/licenses/by/2.0), which permits unrestricted use, distribution, and reproduction in any medium, provided the original work is properly cited. 
prediction error to refine the weights of each expert's contribution to the prediction; the updated weights are used in the next iteration of the algorithm. We contend that by employing the proposed prediction technique, network applications, and protocols that make use of the RTT do not have to measure it as frequently.

As an example application for the proposed RTT estimation approach, we use it to predict TCP's RTT. Through extensive simulations and live experiments, we show that our machine learning technique can adapt to changes in the RTT faster and thus predict its value more accurately than the current exponential weighted moving average (EWMA) technique employed by most versions of TCP. As described in Section 2, TCP uses the RTT estimates to compute its retransmission time-out (RTO) timer [3], which is one of the main timers involved in TCP's error and congestion control. When the RTO expires, the TCP sender considers the corresponding packet to be lost and therefore retransmits it. TCP relies on RTT predictions and measurements in order to set the RTO value properly. In TCP, the RTT is defined as the time interval between when a packet leaves the sender and until the reception, at the sender, of a positive acknowledgment for that packet.

If the RTO is too long, it can lead to long idle waits before the sender reacts to the presumably lost packet. On the other hand, if the RTO is set to be too aggressive (too short), it might expire too often leading to unnecessary retransmissions. Needless to say that setting the RTO is critical for TCP's performance.

We can split the problem of setting TCP's RTO into two parts. The first part is how to predict the RTT of the next packet to be transmitted, and the second is how the predicted RTT can be used to compute the RTO. In this paper, we focus on the first part of the problem, i.e., the prediction of the RTT; the second part of the problem, i.e., setting the RTO, is the focus of future work.

To estimate the RTT, we propose a new approach based on machine learning which will be described in detail in Section 3. Our experimental results show that RTT predictions using the proposed technique are considerably more accurate when compared to TCP's original RTT estimation algorithm and the well-known Eifel [4] timer. We then evaluate how this increased accuracy affects network performance. We do so by running network simulations as well as live experiments. For the latter, we have implemented both our machine learning as well as the Eifel mechanism in the Linux kernel.

The remainder of this paper is organized as follows. Section 2 presents related work, including a brief overview of TCP's original RTT estimation technique. In Section 3, we describe our RTT prediction algorithm. Section 4 presents our experimental methodology, where we describe the scenarios conceived for our simulation studies, discuss simulation parameters, and define metrics for performing evaluations. Sections 5 and 6, respectively, present our results from both simulation and live experiments. Finally, Section 7 concludes the paper and highlights directions for future work.

\section{Related work}

In this section, we present a brief overview of previous work on RTT estimation and later discuss some relevant machine learning applications.

\subsection{TCP RTT estimation}

TCP uses a time-out/retransmission mechanism to recover from lost segments. This time-out value needs to be greater than the current RTT to avoid unnecessary retransmissions; on the other hand, if it is too high, it will cause TCP to wait too long to react to losses and congestion. In Jacobson's well-known work [3], two state variables EstimatedRTT and RTVAR keep the estimate of the next RTT measurement (sampleRT) and the RTT variation, respectively. RTVAR is defined as an estimate of how much EstimatedRT typically deviates from SampleRT. EstimatedRT is updated according to an EWMA given by Equation 1, where $\alpha=\frac{1}{8}$. RTVAR is calculated using Equation 2 which is also an EWMA; this time of the difference between SampleRT and EstimatedRT with gain $\beta$ typically set to $\frac{1}{4}$. Equation 3 sets the new value for the RTO as a function of EstimatedRTT and RTTVAR, where $K$ is usually 4 .

EstimatedRTT $=(1-\alpha) \cdot$ EstimatedRTT $+\alpha \cdot$ SampleRTT

$\operatorname{RTVAR}=(1-\beta) \cdot \mathrm{RTTVAR}+\beta \cdot \mid$ SampleRTT- EstimatedRTT $\mid$

$\mathrm{RTO}=\max ($ EstimatedRTT $+K \cdot$ RTTVAR, 2.ticks $)$

Most current variants of TCP also implement Karn's Algorithm [5], which ignores the SampleRT corresponding to retransmissions. Another consideration is TCP's clock granularity. In several TCP implementations, and also in the simulation tool used in this work, the clock advances in increments of ticks commonly set to $500 \mathrm{~ms}$, and the RTO is bounded by RTO $\min =2 \cdot$ ticks and $\mathrm{RTO}_{\max }=64$. ticks $^{\mathrm{b}}$.

In prior work, a number of approaches have been proposed to estimate TCP's RTT. Trace-driven simulations reported in [6] to evaluate different RTT estimation algorithms show that the performance of the estimators is dominated by their minimum values and is not influenced by the RTT sample rate [6]. This last conclusion was challenged by the Eifel estimation mechanism [4], one of the most cited alternatives to TCP's original RTT estimator; Eifel can be used to estimate the RTT and set the RTO. Eifel's proponents identify several problems with TCP's original RTT estimation algorithm, including the observation that a sudden decrease in RTT causes RTTVAR and consequently the RTO to increase unexpectedlyc. As it 
will become clear from the experimental results presented in Subsection 6.3, our approach is able to follow quite closely any abrupt changes in the RTT and outperforms both TCP's original RTT estimator as well as that of Eifel's.

Another notable adaptive TCP RTT estimator was proposed in [7]. It uses the ratio of previous and current bandwidth to adjust the RTT. In [8], a TCP retransmission time-out algorithm based on recursive weighted median filtering is proposed. Their simulation results show that for Internet traffic with heavy tailed statistics, their method yields tighter RTT bounds than TCP's original RTT computation algorithm. Leung et al. present work that focuses on changing RTO computation and retransmission polices, rather than improving RTT predictions [9].

\subsection{Selected machine learning applications}

Machine learning has been used in a number of other applications. Helmbold et al. use an experts framework algorithm to predict hard-disk drive's idle time and decide when to attempt to save energy by spinning down the disk [10]. For this problem, the cost of making a bad decision (i.e., when spinning the disk down and back up costs more than simply leaving it on) is very well defined since the decision of spinning down the disk does not affect the length of the next idle time.

Unlike the spin-down example, when predicting the RTT, every prediction causes the next RTT to be set to a different value and that influences every event that happens thereafter. Thus, the problem of defining the cost of a bad RTT prediction is not as straightforward. Our solution to this problem is discussed in Section 3.

Moreover, in the spin-down cost problem, the traces used in the evaluation were 'off-line' traces, i.e., traces captured from live runs and later on used as input to the algorithms being evaluated. In the case of the spin-down problem, there is no problem in using off-line traces since the algorithm's estimations do not influence the outcome of the next measurement. In the case of TCP RTT estimation, however, as previously discussed, since the RTT estimations influence TCP timers and these timers affect the outcome of the next RTT measurement, off-line traces can be used to set and tune parameters of the algorithm, but they are not suitable for evaluating the performance of the system. The work presented in $[8,9,11,12]$ on RTT estimation compares their solution against TCP's original RTT estimation algorithm, but they base their evaluation on off-line traces.

Machine learning techniques have not been commonly employed to address network performance issues. The work described in [13] is a notable exception and proposes the use of the stochastic estimator learning algorithm (SELA) to address the call admission control (CAC) problem in the context of asynchronous transfer mode (ATM) networks. Their goal was to predict in 'real time' if a call request should be accepted or not for various types of traffic sources. Simulation results show the statistical gain exhibited by the proposed approach compared to other CAC schemes. Another SELA-based approach, this time, applied to QoS routing in hierarchical ATM networks was proposed in [14]. In this approach, learning algorithms operating at various network switches determine how the traffic should be routed based on current network conditions. In the context of WiMax networks, a cross-layer design approach between the transport and physical layers for TCP throughput adaptation was introduced in [15]. The proposed approach uses adaptive coding and modulation (ACM) schemes.

Mirza et al. propose a throughput estimation tool based on support vector regression modeling [16]. It predicts throughput based on multiple real-value input features. However, to the best of our knowledge, to date, no attempt to use on-line learning algorithms to predict network conditions has been reported.

\section{Proposed approach}

In this section, we present the fixed-share experts algorithm as a generic solution for on-line prediction. Later, we describe its application to the problem of predicting the RTT of a TCP connection.

\subsection{The fixed-share experts algorithm}

Our RTT prediction algorithm is based on the fixedshare experts algorithm [2] which uses 'on-line learning' based on the predictions of a set of fixed experts denoted by $\left\{x_{1}, \ldots, x_{N}\right\}$. In on-line learning, the learning process happens in trials. Figure 1 illustrates these trials and the algorithm itself. Under the fixed-share experts algorithm, at every trial $t$, the algorithm receives the predictions $x_{i} \forall i \in\{1, \ldots, N\}$ from a total of $N$ experts and uses them to output a master prediction $\hat{y}_{t}$. After trial $t$ is completed, the 'ground truth' value $y_{t}$ becomes known and is used to compute the estimation error based on a loss function, $L$. The estimation error computed at trial $t$ for every expert $i$ is thus given by $L_{i, t}\left(x_{i}, y_{t}\right)$ which is used to update a set of weights. The next prediction for trial $t+1$ is calculated using this new set of weights denoted by $W_{t+1, i}=$ $\left\{w_{t+1,1}, \ldots, w_{t+1, i}, \ldots, w_{t+1, N}\right\}$.

The weight $w_{t, i}$ should be interpreted as a measurement of the confidence in the quality of the $i$ th expert's prediction at the start of trial $t$. In the initialization of the algorithm, we make $w_{1, i}=\frac{1}{N}, \forall i \in\{1, \ldots, N\}$. The algorithm updates the experts' weights at every trial after computing the loss at trial $t$ by multiplying the weight of the $i$ th expert by $e^{-\eta L_{i, t}\left(x_{i}, y_{t}\right)}$. The learning rate $\eta$ is used to determine how fast the updates will take effect, dictating how rapidly the weights of misleading experts will be reduced. 


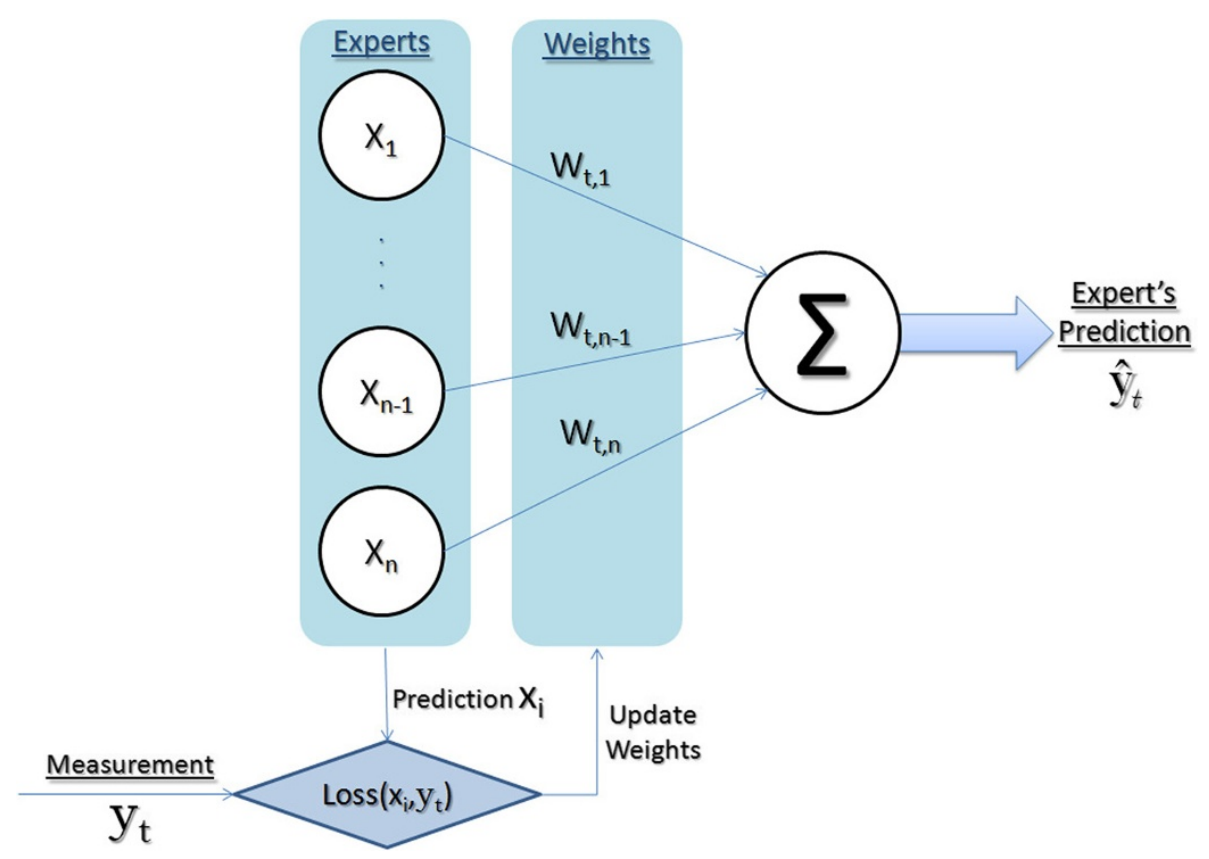

Figure 1 Graphical representation of the general fixed-share experts algorithm for RTT estimation.

After updating the weights, the algorithm also 'shares' some of the weight of each expert among other experts. Thus, an expert who is performing poorly and had its weight severely compromised can quickly regain influence in the master prediction once it starts predicting well again. The amount of sharing can be changed by using the $\alpha$ parameter, called the sharing rate. This allows the algorithm to adapt to 'bursty' behavior. Indeed, in Section 5, we use, among others, bursty traffic scenarios to evaluate the performance of our algorithm.

Algorithm 1 summarizes the steps involved in our fixedshare experts algorithm. The first line in the algorithm summarizes the algorithm's parameters, namely: (1) the learning rate $\eta$, which we define as a positive real number, and (2) the sharing rate $\alpha$, a real number between zero and one that dictates the percentage of the weights shared at every trial. In our approach, expert weights are initialized uniformly, which is what is described in the 'Initialization' step of Algorithm 1. The basic four steps of our fixed-share experts algorithm are also summarized in Algorithm 1 (and in Figure 1). In step 1, the prediction defined as a the weighted average over the individual predictions $x_{i}$ of every $i$ th expert is computed. The loss function is computed in step 2 and is used in step 3 to penalize and decrease the weights of the experts that are not performing well, and in step 4, experts share their weight based on the sharing rate $\alpha$.

In [2], the basic version of the experts framework is presented along with bounds for different loss functions. The algorithm is also analyzed for different prediction functions, including the weighted averaging we use. The implementation described in this paper, with the intermediate pool variable, costs $O(1)$ time per expert per trial.

\subsection{Applying experts to TCP's RTT prediction}

To apply the proposed algorithm to TCP's RTT-prediction problem, the experts predictions $x_{i}$ shown in Algorithm 1 serve as predictions for the next RTT measured. $y_{t}$ is the RTT value at the present trial, equivalent to the SampleRT in the original TCP RTT estimator. $\hat{y}_{t}$ is the output of the algorithm, or in other words, the RTT prediction itself, equivalent to the EstimatedRT in the original TCP RTT predictor, mentioned in Section 2.

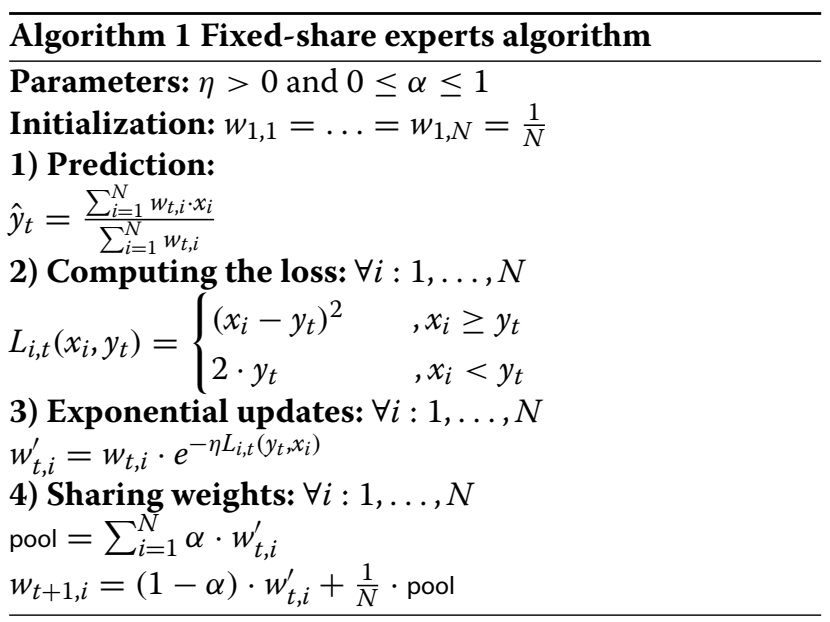


We want the loss function $L_{i, t}\left(x_{i}, y_{t}\right)$ to reflect the real cost of making wrong predictions. In our implementation (see Algorithm 1), the loss function has different penalties for overshooting and undershooting the RTT estimate as they have different impact on the system's behavior and performance. An underestimate of the RTT will result in an RTO computation that is less than the next measured RTT, causing unnecessary timeouts and retransmissions. We thus employ the following policies: if the measured RTT $y_{t}$ is higher than the expert's prediction $x_{i}$, then it means that this expert is contributing to a spurious timeout and should be penalized more than other experts that overshoot within a given threshold. Big over-shooters are more severely penalized. The challenge here is identifying the appropriate cost for miss-predicting the RTT. The cost could be simply the difference between prediction and measurement, or a factor thereof. Exploring other loss functions for the TCP RTT estimation problem is the subject of future work.

Setting the value $x_{i}$ of the experts is referred to as setting the experts spacing. To space the experts is to determine the experts' values and their distribution within the prediction domain. When predicting RTTs, the experts should be spaced between $R T_{\min }$ and $R T_{\max }$, defined in some TCP implementations (including the one in the network simulator we used in our experimental evaluation) to be 1 and 128 ticks, respectively. Based on observations of several RTT datasets, we concluded that the majority of the RTT measurements are concentrated in the lower part of this interval. For that reason, we found that spacing the experts exponentially in that interval, instead of uniformly (or linearly), leads to better predictions. The exponential function used in our implementation of the Fixed-Share Experts approach is $x_{i}=\mathrm{R} \Pi_{\min }+\mathrm{R} \Pi_{\max } \cdot 2^{\frac{(i-N)}{4}}$. The $\frac{1}{4}$ multiplicative factor in the exponent of the spacing function was experimentally chosen to smooth out its growth. This increases the difference between the experts and generates diversity among them, which increases predictions' granularity and accuracy.

Another consideration is that the algorithm, as stated in Algorithm 1, will continually reduce the experts' weights towards zero. Thus, in order to avoid underflow issues in our implementation, we periodically rescale the weights. Different versions of the multiplicative weight algorithmic family, including a mixing past approach that also mixes weights from past trials are discussed in [17]. However, the mixing past approach incurs higher space and time costs and thus was not considered in our work. Another sharing scheme known as variable-sharing was also considered in preliminary experiments, where the amount of shared weights to each expert was dependent of their individual losses. However, the additional complexity and cost of this scheme outweighed its benefits in terms of RTT estimation accuracy.

\section{Experimental methodology}

We conduct simulations using the QualNet [18] network simulation platform. In all experiments, unless stated otherwise, the simulation area is $1,500 \mathrm{~m} \times 1,000 \mathrm{~m}$ and the simulated time is $25 \mathrm{~min}$. The routing protocol used was AODV [19], and the medium access and physical layers defined at the IEEE 802.11.b [20] standard was used. The transmission radius was set to $100 \mathrm{~m}$ for all the nodes, which are initially placed in the simulation area uniformly distributed. On mobile scenarios, nodes move according to the random way point (RWP) mobility regime with $0 \mathrm{~s}$ of pause time and speed ranging between $[1,50] \mathrm{m} / \mathrm{s}$. All nodes run file transmission protocol (FTP) applications to generate the TCP flows; TCP buffer size is the default TCP buffer size set to 16,384 bytes, and packet size is fixed at 512 bytes. For the reader convenience, we summarize the simulation parameters for all simulation scenarios in Table 1 . The value $n a$ is attributed to a given parameter in this table when it is not applicable. These and other simulation parameters, such as traffic patterns and number of flows will be discussed further in the following sections in a per-scenario basis.

In Section 5, we present results for a total of five simulation scenarios, which are summarized, along with their parameters, in Table 1. The goal of having a variety of scenarios is to subject the proposed RTT estimation approach to a wide range of network conditions. Scenarios I and II represent ad hoc mobile networks composed of 20 and 10 nodes, respectively. These two scenarios differ from each other only in terms of node density. Scenario III is a static wireless network composed of 20 nodes uniformly distributed over the simulated network area. Scenario IV is also a 20-node mobile network, but the traffic pattern is different from scenarios I and II. In scenario IV, we also vary the mobility of the network by varying the nodes' average speed. Finally, scenario V is a wired network composed of eight nodes, four on each side of a bottleneck link.

It is important to highlight that the mobile scenarios employed in our evaluation were used to evaluate the proposed RTT estimation technique under conditions that cause high variability and randomness in the network and thus in the RTT values. Following the RWP mobility regime, nodes move randomly causing data paths to break and new ones to be created which then results in high variability of the RTT. Our goal was then to ensure that our RTT estimator is able to adjust to high variability conditions and yield accurate RTT estimates.

We present our results in Section 5 using a number of performance metrics defined as follows:

- Mean error on RTT prediction: absolute difference between the RTT prediction $\hat{y}_{t}$ and the measured $\mathrm{RTT} y_{t}$ at trial $t$, averaged over all trials, defined as: 
Table 1 Simulation parameters for each simulation scenario

\begin{tabular}{|c|c|c|c|c|c|}
\hline Parameter & Scenario I & Scenario II & Scenario III & Scenario IV & Scenario V \\
\hline Routing & AODV & AODV & AODV & AODV & fixed \\
\hline Mobility & RWP & RWP & none & RWP & na \\
\hline $\mathrm{MAC} / \mathrm{PHY}$ & 802.11.b & 802.11.b & 802.11.b & 802.11.b & 802.3 \\
\hline Speed $[\min , \max ](\mathrm{m} / \mathrm{s})$ & {$[1,50]$} & {$[1,50]$} & 0 & {$[1,10],[20,30],[40,50]$} & na \\
\hline Pause (sec) & 0 & 0 & 0 & 0 & na \\
\hline Area (meters $\times$ meters) & $1,500 \times 1,000$ & $1,500 \times 1,000$ & $1,500 \times 1,000$ & $1,500 \times 1,000$ & na \\
\hline Duration (min) & 25 & 25 & 25 & 90 & 90 \\
\hline Number of nodes & 20 & 10 & 20 & 20 & 8 \\
\hline
\end{tabular}

$\frac{1}{T} \sum_{t=1}^{T}\left|\hat{y}_{t}-y_{t}\right|$. In the simulations, RTT is measured in 'ticks' of $500 \mathrm{~ms}$. This value is equal to 4 $\mathrm{ms}$ in the real live experiments described in Section 6. The mean RTT prediction error metric is thus, also given in 'ticks'.

- Average congestion window size (cwnd): the size in bytes of the congestion window at the TCP sender, averaged over all prediction trials, defined as: $\frac{1}{T} \sum_{t=1}^{T} c w n d_{t}$, where $c w n d_{t}$ is the congestion window size measured at trial $t$.

- Delivery ratio: ratio between total data packets received by the destination and data packets sent at the source, computed for every flow and averaged over all flows.

- Percentage of retransmitted packets: ratio between retransmitted data packets and total data packets sent at every flow, averaged over all flows.

- Goodput: total number of useful (data) packets received at the application layer divided by the total duration of the flow and averaged for every flow, giving a ratio of packets per second.

Following, we discuss the impact of the parameters in the accuracy of the proposed RTT prediction algorithm and justify the choice of values set to these parameters in our simulation evaluations.

\subsection{Impact of experts framework parameters $(N, \eta, \alpha)$}

We experimented with several combinations of the fixedshare algorithm parameters. The number $N$ of experts affects the granularity over the range of values the RTT can assume. In our experiments, $N>100$ had no major impact on the prediction accuracy.

The learning rate $\eta$ is responsible for how fast the experts are penalized for a given loss. We want to avoid values of $\eta$ that are too low since it increases the algorithm's convergence time; conversely, if $\eta$ is too high, it forces the expert's weights toward zero too quickly. If this is the case, then as weight rescaling kicks in, the algorithm assigns similar weights to all experts, making the algorithm's master prediction fluctuate undesirably around the mean value of the experts' guesses. We chose a learning rate in the interval $1.7<\eta<2.5$ as it provides good prediction results for all the scenarios tested.

When sharing is not enabled, i.e., $\alpha=0$, the outcome of the algorithm is given only by decreasing exponential updates, making it harder for the algorithm to follow abrupt changes in the RTT measurements: experts that experience prolonged poor performance lack influence because their weights have become too depreciated. In this case, it would take more trials so that these experts start gaining greater importance in the master prediction. Enabling full sharing $(\alpha=1)$, similar weights are assigned to every expert, and the master prediction fluctuates close to a mean value among the experts guesses.

We present in more detail the simulation scenarios in the next section, highlighting the goals for every evaluation and discussing obtained results. Following our observations, in all results reported hereafter concerning our proposed approach, we use $N=100, \eta=2$ and $\alpha=0.08$.

\section{Simulation results}

In this section, we discuss simulation results obtained by applying the fixed-share experts framework to estimate TCP's RTT and help set TCP's RTO timer. We compare our results against TCP's original RTT and RTO computation algorithm by Jacobson [3]. We evaluate the RTT prediction quality and how it impacts the previously defined performance metrics. We consider different scenarios by varying network density, mobility, and traffic load. Both wireless as well as wired networks are used in our evaluation.

In all graphs presented below, each data point is computed as the average over 24 simulation runs with a confidence level of $90 \%$.

\subsection{Scenario I - mobile scenario ( 20 nodes)}

First, we considered a mobile ad hoc network (MANET) composed of 20 nodes. The goal of this scenario is to evaluate the performance of the RTT prediction algorithms when routes in the network change widely. In other words, we want to show the algorithm's response 
to RTT fluctuations. For that reason, we also varied the number of TCP flows during the simulations to change network load and congestion levels. We evaluated scenarios with number of concurrent flows equal to $3,7,17,34$, 68,100 , and 130. Although flows were evenly distributed among nodes, they started at random times during the experiments and their sizes varied from 1,000 to 100,000 packets.

Figure 2 shows the mean differences between the predictions of the experts framework and Jacobson's algorithms when compared to the real RTT measurements. We observe that the experts framework improves RTT estimation accuracy considerably as the load in the network increases. This leads to more accurate setting of the RTO timer, which, in turn, reduces the relative number of packets retransmitted significantly, especially under heavy traffic conditions. As shown in Figure 3, retransmissions are reduced substantially (by as much as over $30 \%$ ) without decreasing the number of packets sent. Note also the improvement in the goodput, i.e., total number of packets received (Figure 4 ).

Our use of the experts framework not only improved the RTT predictions, thereby avoiding unnecessary retransmissions, but also avoided unnecessary triggering of congestion-control mechanisms. Consequently, TCP's congestion window ( $c w n d$ ) is higher on average; this behavior is shown in Figure 5, which plots the average cwnd for the experts framework and Jacobson's algorithms over the duration of the whole simulation for different congestion scenarios, i.e., different number of TCP flows. We can observe how the gap between the two approaches increases with increasing congestion conditions. This indicates that the experts framework is able to better shield TCP's congestion control from wide RTT fluctuations.

Figure 6 plots the average cwnd over the whole simulation time for 3, 34, and 130 flows. Each point in this plot is the mean cwnd over 24 simulation runs considering all nodes averaged over a 20 -s time window. These plots corroborate the results shown in Figure 5, i.e., that the experts framework yields, on average, higher cwnd. We observe from Figures 7 and 4 that the proposed experts framework is also able to improve both the delivery ratio and goodput, which we define here as the absolute number of packets successfully delivered at the destination over the course of the simulation.

\subsection{Scenario II - mobile scenario ( 10 nodes)}

In this scenario, we try to subject the network to varying network conditions in order to produce higher RTT variations. We accomplish that by running the same scenario, but now decreasing the density of the network, which includes only ten mobile nodes. The objective is to cause more frequent route changes which would result in more frequent and wider RTT variations. For example, in scenario I, the mean RTT variance for 34 and 100 flows are 0.4763 and 0.5501 ticks, respectively. For scenario II, with only ten nodes, the mean RTT variance for the same congestion scenarios are 0.7878 and 0.9315 ticks, respectively. Except for the reduced number of nodes, all the other parameters for this scenario are the same as before.

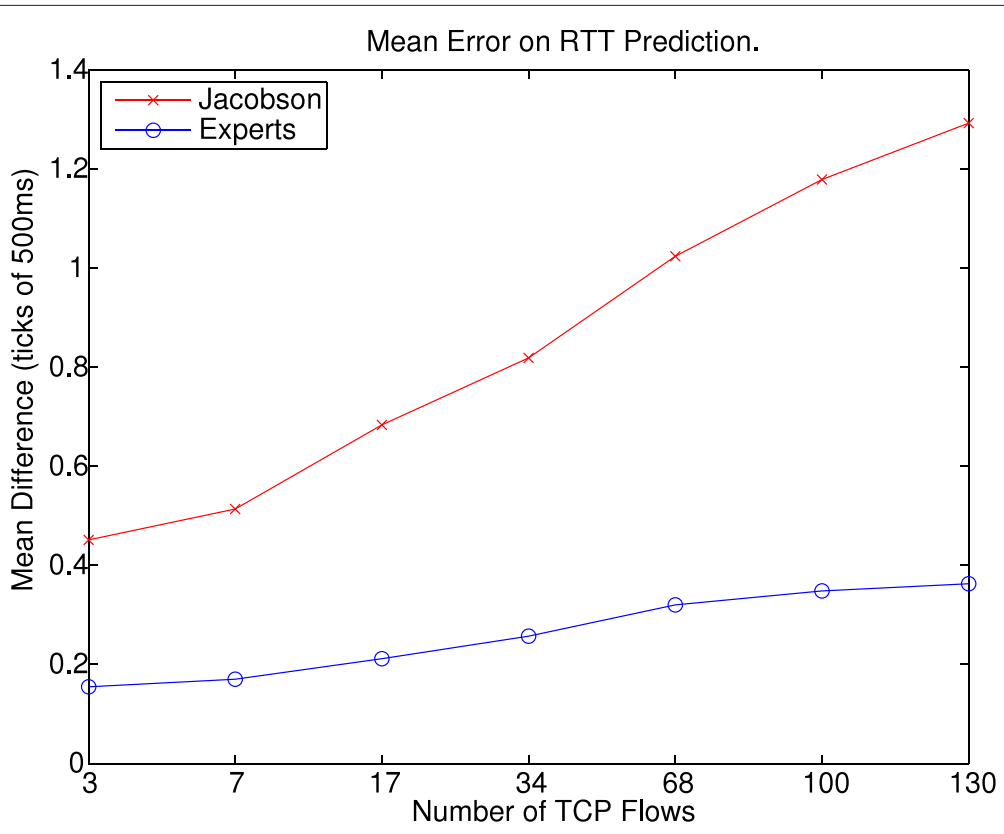

Figure 2 Mean absolute difference between predicted and measured RTT on a MANET with 20 nodes. 


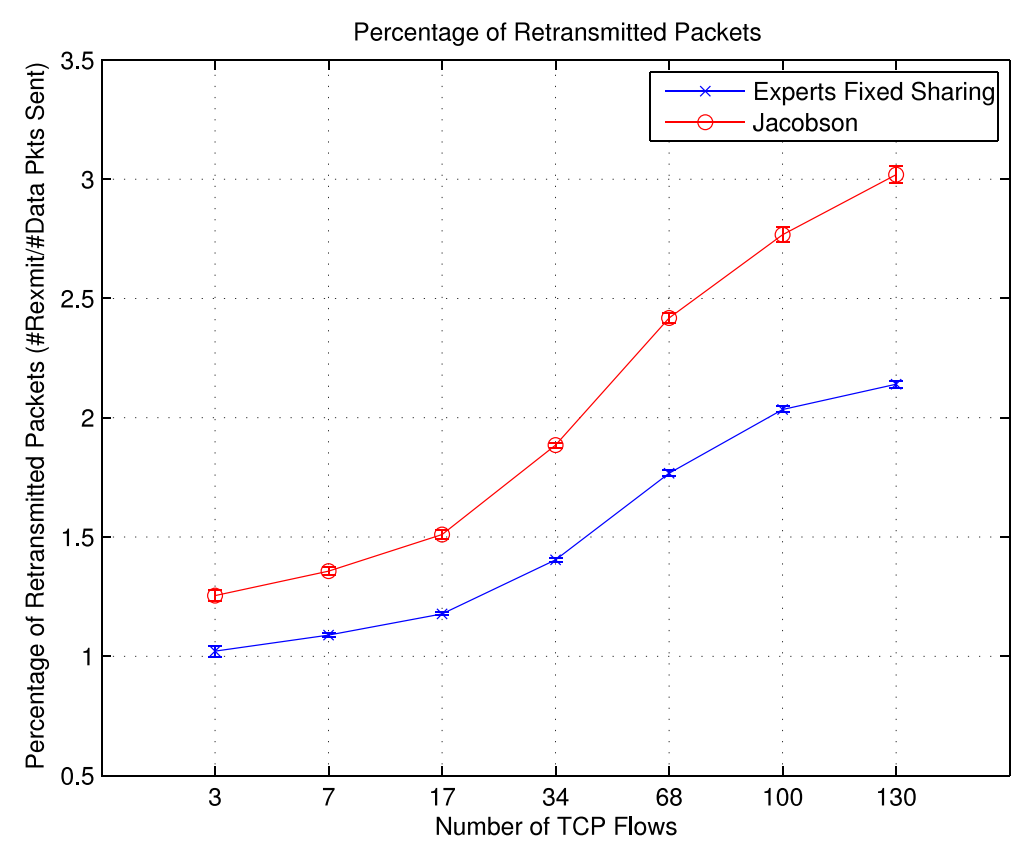

Figure 3 Number of retransmitted packets in relation to the total number of packets transmitted on a 20-node MANET.

In Figure 8, we can compare the accuracy of the predictions for both algorithms. Similar to the previous scenario, as congestion increases with the number of flows, so does the variability of the RTT measurements, also increasing the mean error in the prediction. For the same number of flows, we observe how the mean error increases from scenario I (Figure 2) to scenario II (Figure 8) due to the increase in the variation of the RTT. Network performance metrics, such as number of retransmitted packets (Figure 9) and delivery ratio (Figure 10) exhibit trends similar to scenario I. Similar behavior is also observed for cwnd (Figure 11), delivery ratio (Figure 10), ratio of packets retransmitted (Figure 9), and goodput in (Figure 12). As in scenario I, we also observe that, as traffic load increases, the difference between the algorithms also increases for all the metrics.

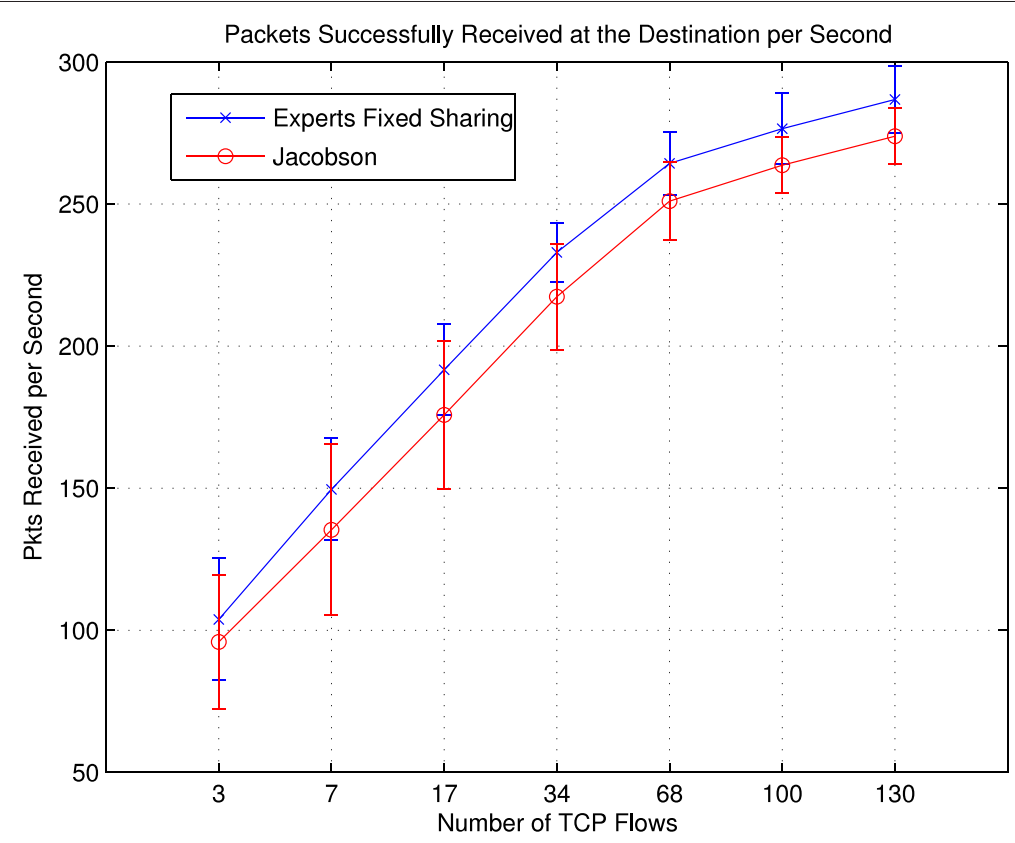

Figure 4 Total number of packets delivered over the duration of the simulation for a 20-node MANET. 


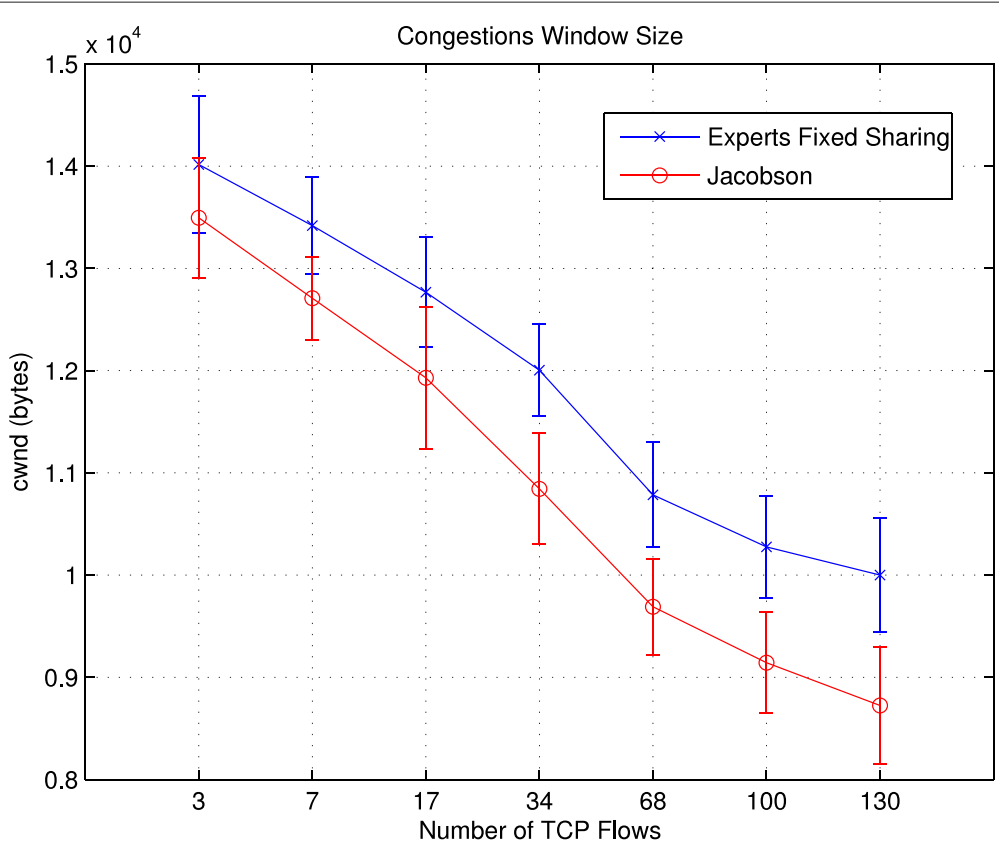

Figure 5 TCP's cwnd for a 20-node MANET for different traffic loads.

\subsection{Scenario III - stationary network}

Our goal in this experiment is to isolate the effect of traffic load on the performance of the proposed RTT estimator. Therefore, we factor out node mobility and consider a wireless ad hoc network where all nodes are stationary. We varied traffic load the same way we did for scenario I.

Figure 13 shows the accuracy of the prediction algorithms. Like in previous scenarios, increasing traffic load degrades the performance of the algorithms, although this degradation is much more pronounced for Jacobson's RTT predictor. This figure shows that the error of the original TCP RTT predictor can get up to around $130 \%$ larger than when using our proposed machine learning approach. For example, in Figure 13, we report an average error of 0.63 ticks for the experts framework, against 1.54 ticks for the original TCP estimator.

Moreover, when comparing the accuracy results for the stationary scenario with the mobile scenario with 20

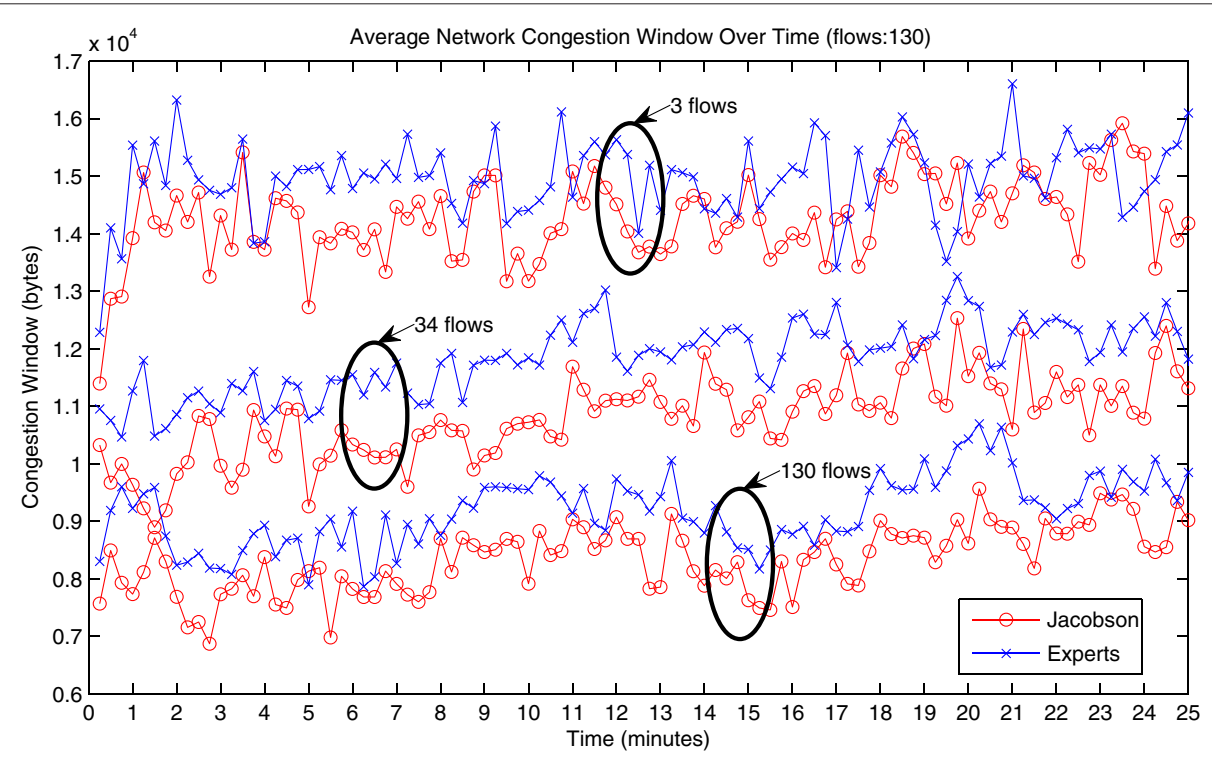

Figure 6 TCP's cwnd over time for 3, 34, and 130 concurrent flows in a 20-node MANET. 


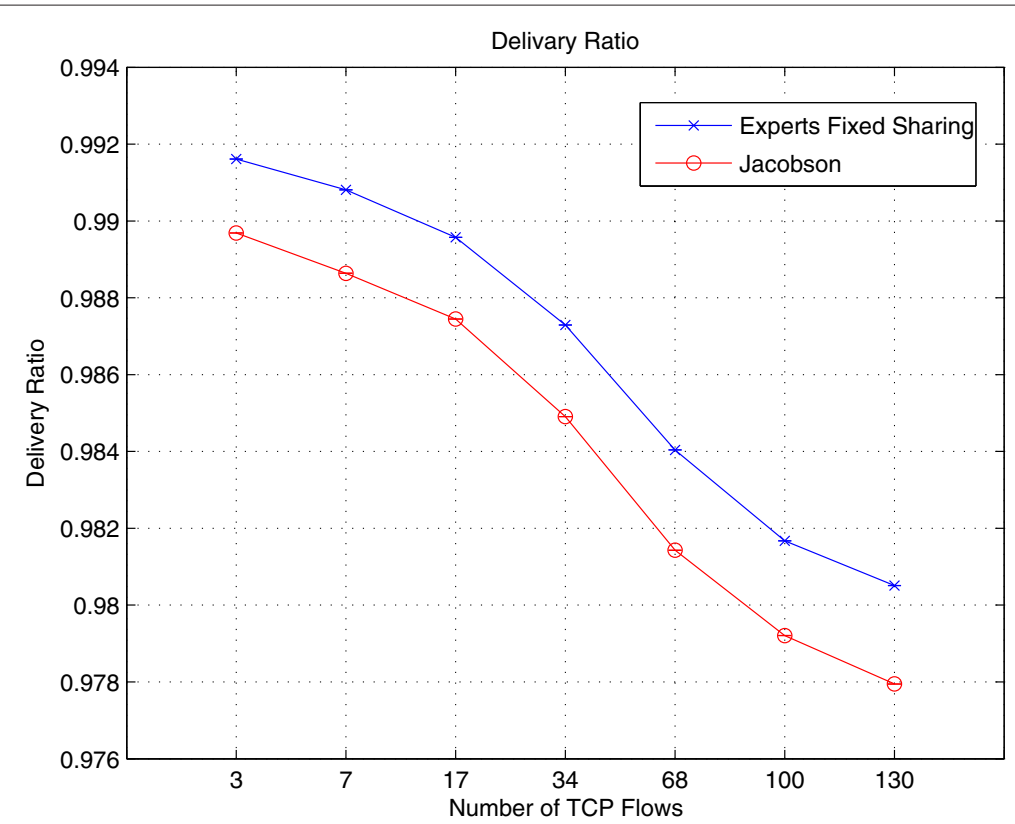

Figure 7 Packet delivery ratio in a 20-node MANET.

nodes (Figure 2), it is possible to notice that the stationary scenario exhibits higher average estimation error for both algorithms. Figure 14 shows the average queue length in bytes for both mobile and stationary scenarios, for different number of flows. We observe that the average queue length for the mobile scenario is much lower. This can be explained by the fact that, in the static scenario, since routes between source and destination are less volatile, queues build up as the traffic load increases and result in larger RTT fluctuations. This is especially the case of nodes that are carrying traffic that belong to multiple flows.

In the stationary scenario, the number of retransmitted packets is lower when using the proposed experts framework approach when compared against the number of retransmissions resulting from Jacobson's algorithm, as we can see in Figure 15. When compared to the mobile

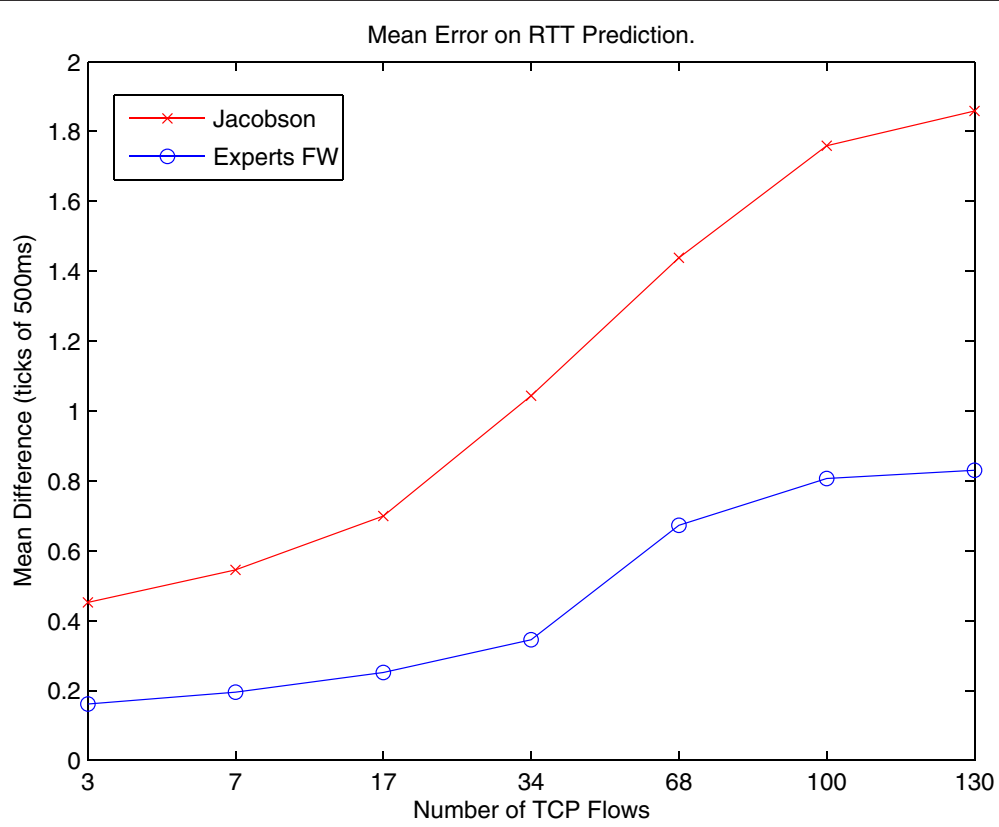

Figure 8 Mean absolute error between the predictions and the measured RTT for a 10-node MANET. 


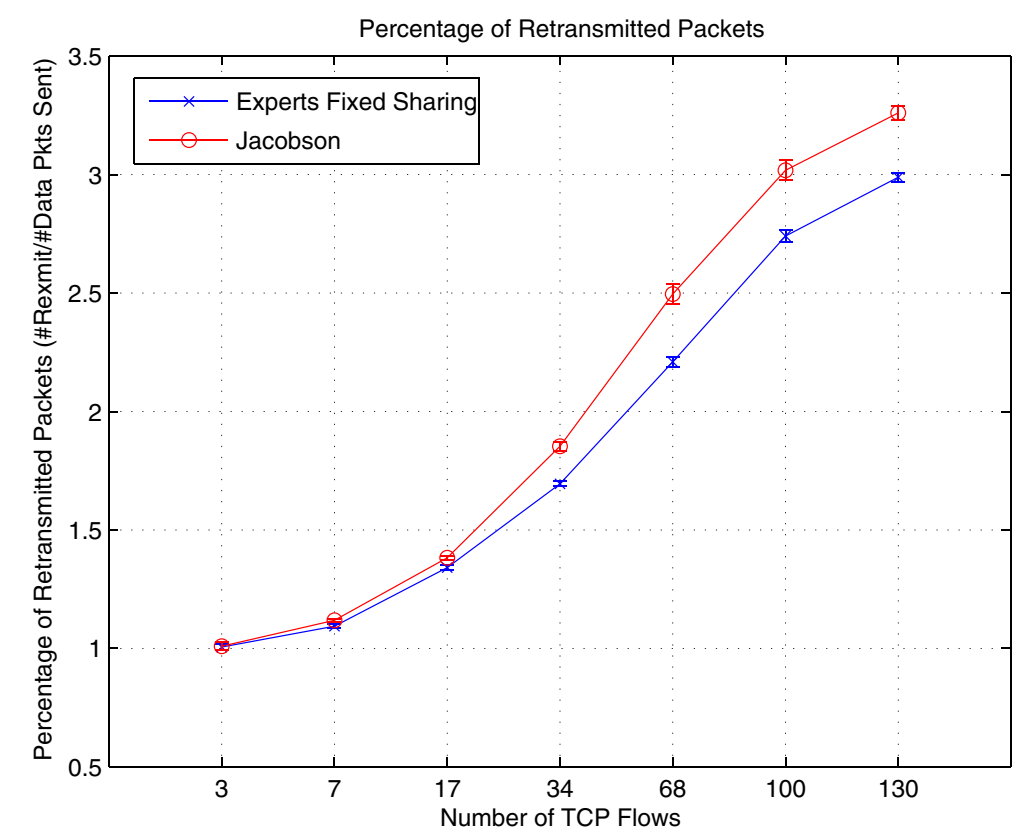

Figure 9 Number of retransmitted packets in relation to the total number of packets transmitted for a 10-node MANET.

scenario, the number of retransmissions is lower for both algorithms since, in the stationary scenario, there are fewer losses due to route failure. Figure 16 shows the average cwnd size for different congestion levels. Once again, it is possible to see improvement in this metric when applying our proposed approach. However, with the increase in the RTT fluctuations in this scenario, as mentioned before, the occurrence of spurious timeouts may also occur. Thus, we experienced in this scenario a higher variability of the cwnd as seen by the larger confidence interval exhibited when comparing it to the cwnd results in Scenario I (Figure 5).

\subsection{Scenario IV - bursty traffic}

In this scenario, we subject the network to bursty traffic loads as a way to evaluate the performance of the proposed

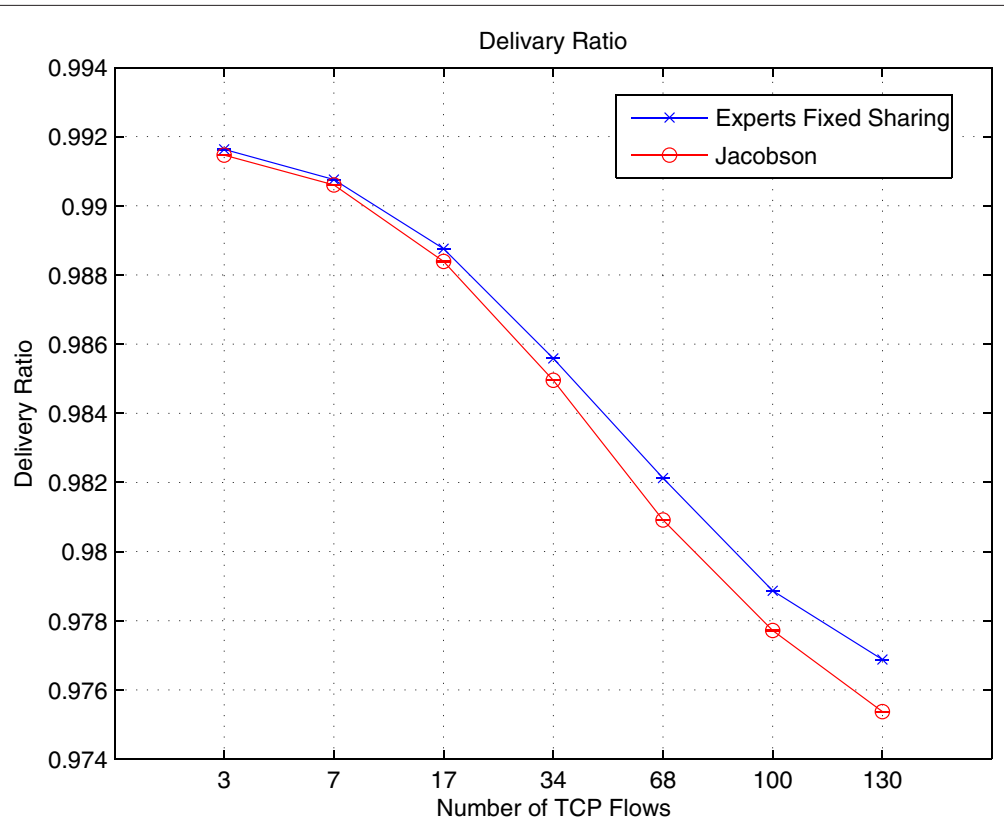

Figure 10 Packet delivery ratio for a 10-node MANET. 


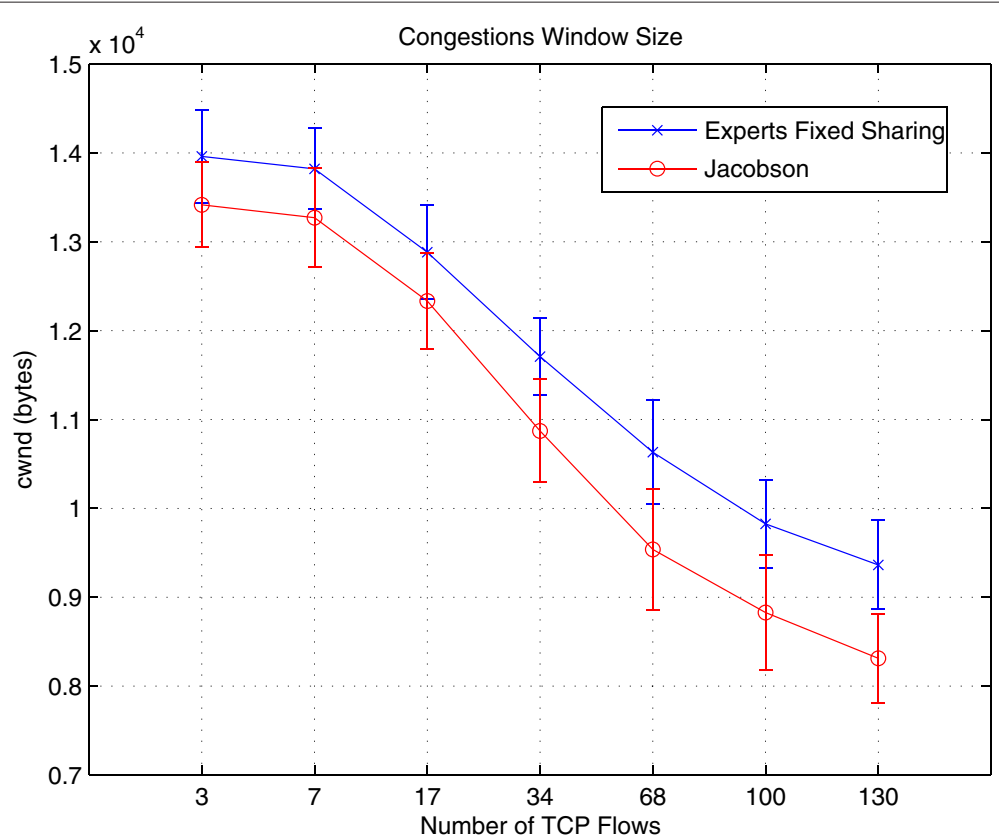

Figure 11 TCP cwnd for a 10-node MANET with different traffic loads.

RTT estimation strategy as traffic load fluctuates. The results shown here reflect the simulation of a network with 20 mobile nodes in which every node starts a TCP flow of 1,000 packets every $200 \mathrm{~s}$; this happens throughout the 90 min of simulation. Thus, nodes would transmit for a while and then remain silent until the next cycle of $200 \mathrm{~s}$. We also vary the speed of the nodes between $(1,10)$,
$(20,30)$, and $(40,50) \mathrm{m} / \mathrm{s}$, which allows average speeds of 5,25 , and $45 \mathrm{~m} / \mathrm{s}$, respectively.

In relation to previous scenarios, the experts framework yields higher performance improvement when compared to Jacobson's algorithm due to the fact that it is able to adapt to RTT fluctuations faster. This is attributed to the weight sharing feature of the experts. This performance

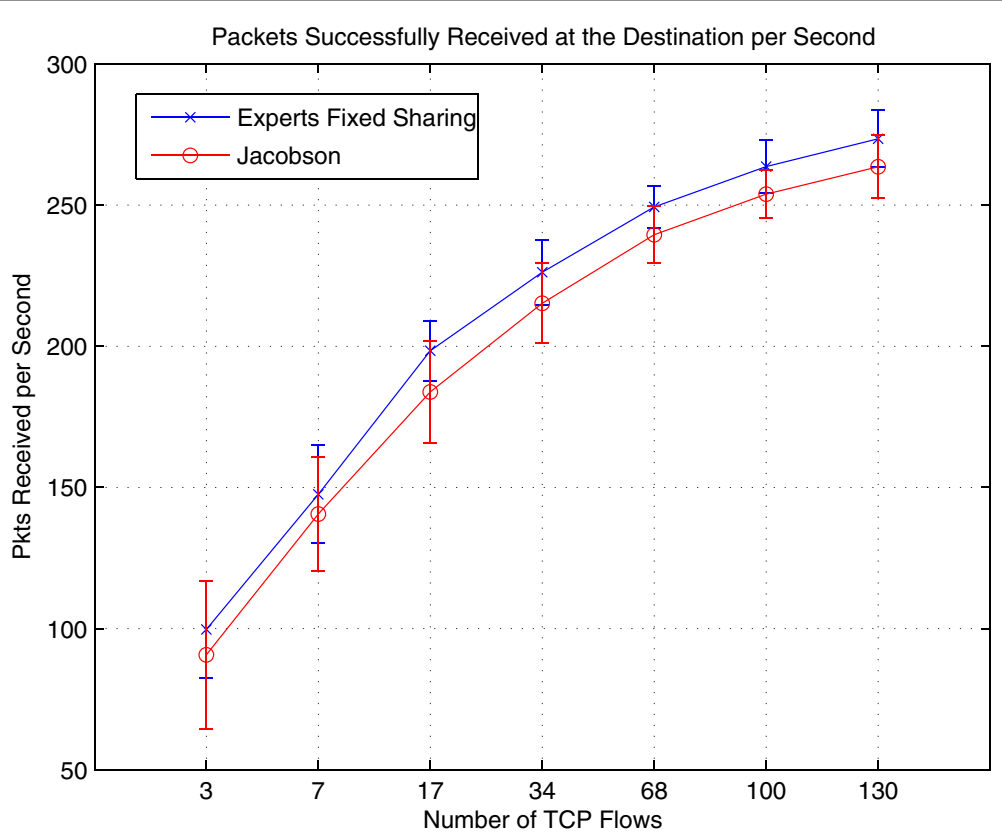

Figure 12 Total number of packets delivered over the duration of the simulation for a 10-node MANET. 


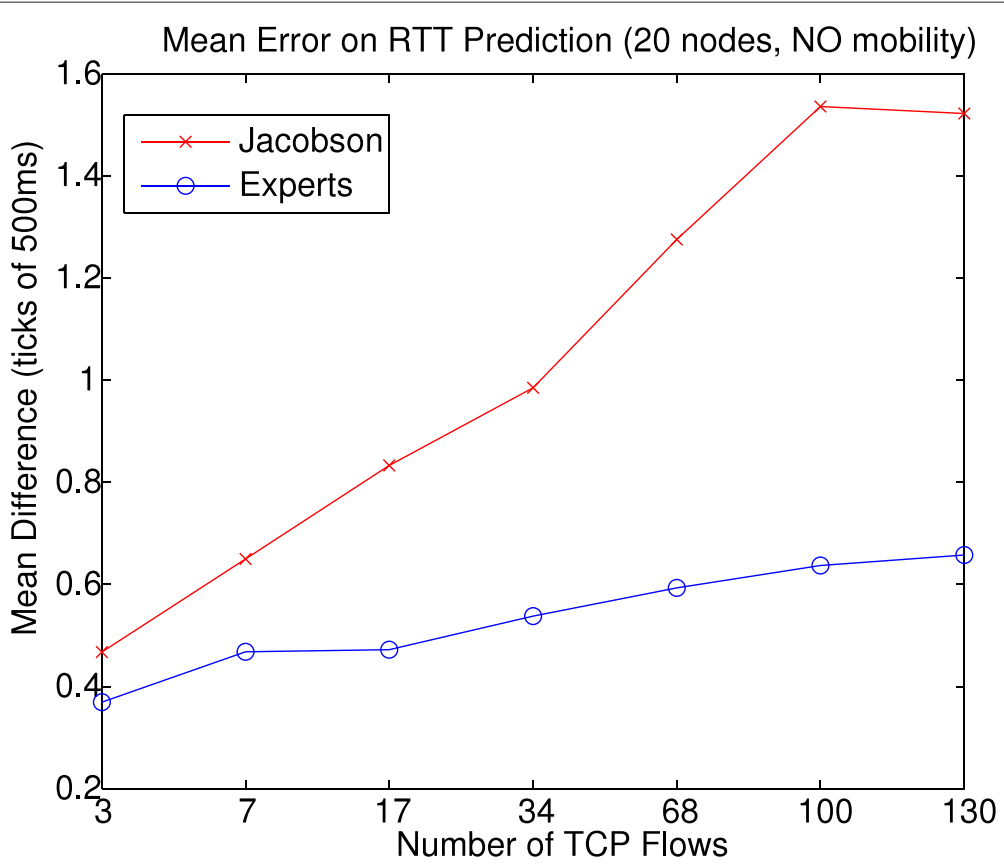

Figure 13 Mean error between the predictions and the measured RTT for the stationary network.

improvement becomes evident when looking at the plot of the mean absolute error in Figure 17. In this figure, the difference between the algorithms prediction errors can vary from $100 \%$ for $25 \mathrm{~m} / \mathrm{s}$ average speed, up to $200 \%$ for $5 \mathrm{~m} / \mathrm{s}$ average speed. We can also observe that the impact of different levels of mobility are also different for both algorithms. While Jacobson's RTT predictor appears to present strong variation with the mobility level, the proposed experts algorithm presents little variation when changing the speed of the nodes. This behavior can be also explained by the fact that the experts framework is able to adapt quickly to abrupt RTT variations because of the sharing mechanism. The moving average applied in Jacobson's algorithm, on the other hand, is not able to respond as fast.

Since the measured RTT fluctuations for this scenario are much greater, the mean prediction error in this case is larger than in the previous scenarios. We also report

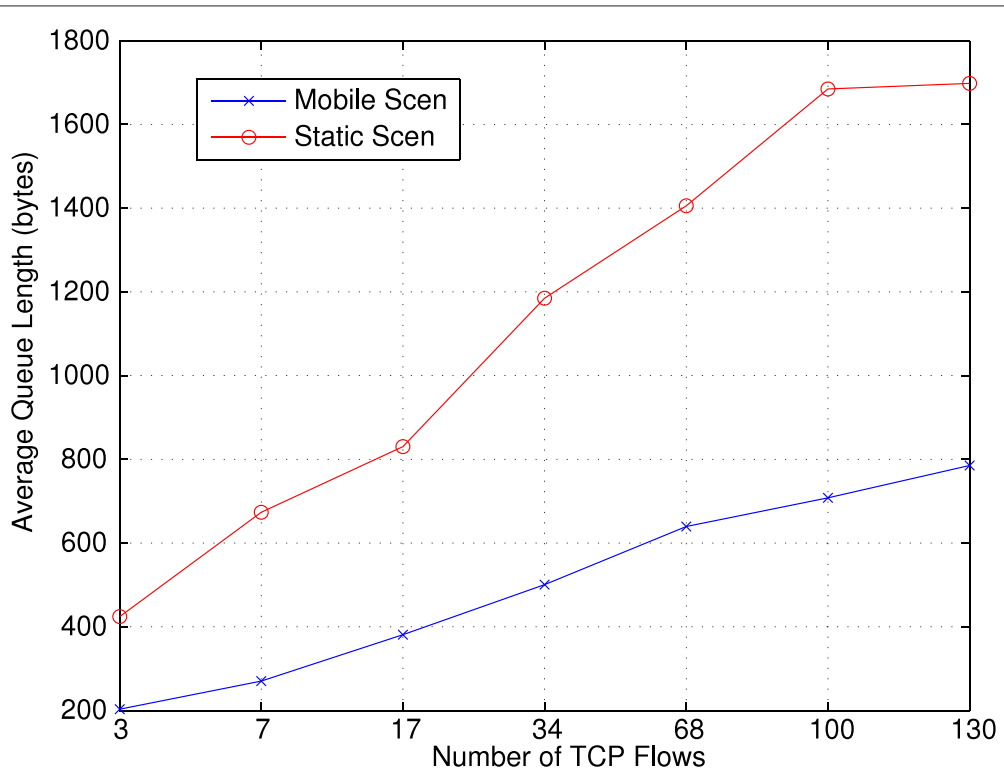

Figure 14 Average queue length in bytes for both mobile and stationary scenarios. 


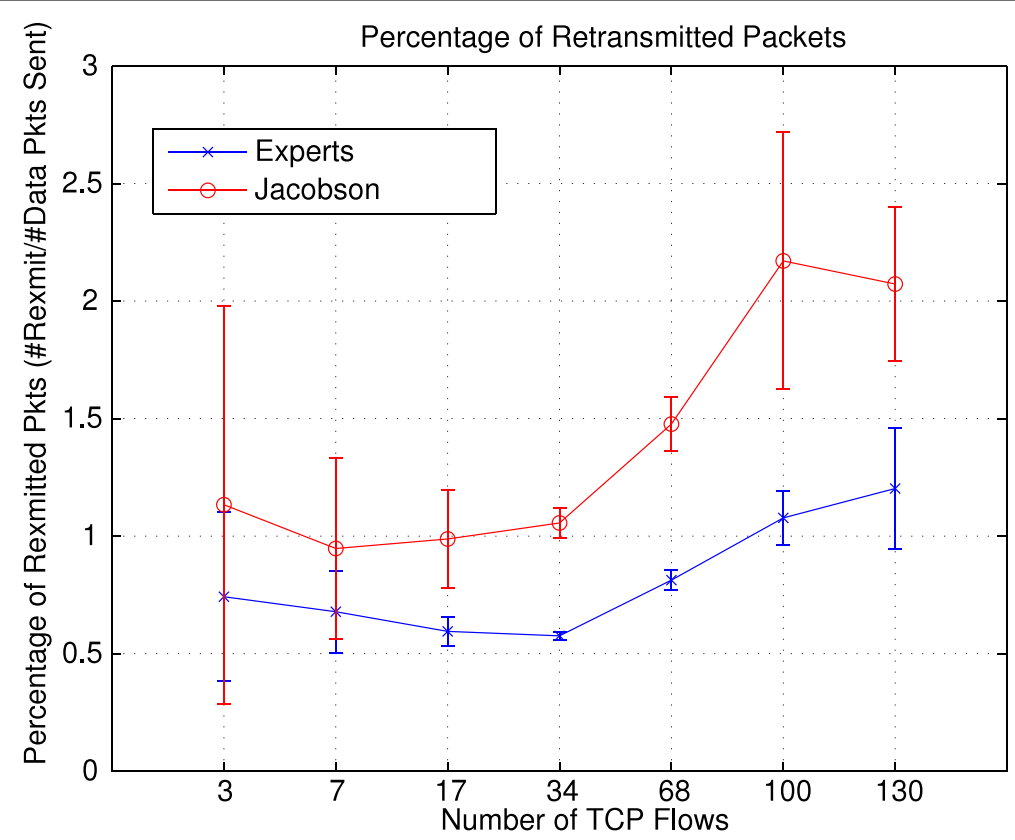

Figure 15 The number of retransmitted packets in relation to the total number of packets transmitted for both algorithms for the stationary scenario.

for this scenario a much lower number of retransmitted packets (Figure 18). It is also possible to observe an improvement in the other performance parameters, i.e., TCP's cwnd, yielding higher goodput (Figure 19) when using our experts framework approach. Finally, Figure 20 shows the improvement in delivery ratio for this scenario.
It is also worth noting the interesting behavior present in the plots for all the reported network performance metrics, where for lower speeds, these metrics reflect better network performance (i.e., lower number of retransmitted packets, higher goodput and higher delivery ratio), since the routing paths do not change as frequently. This incur

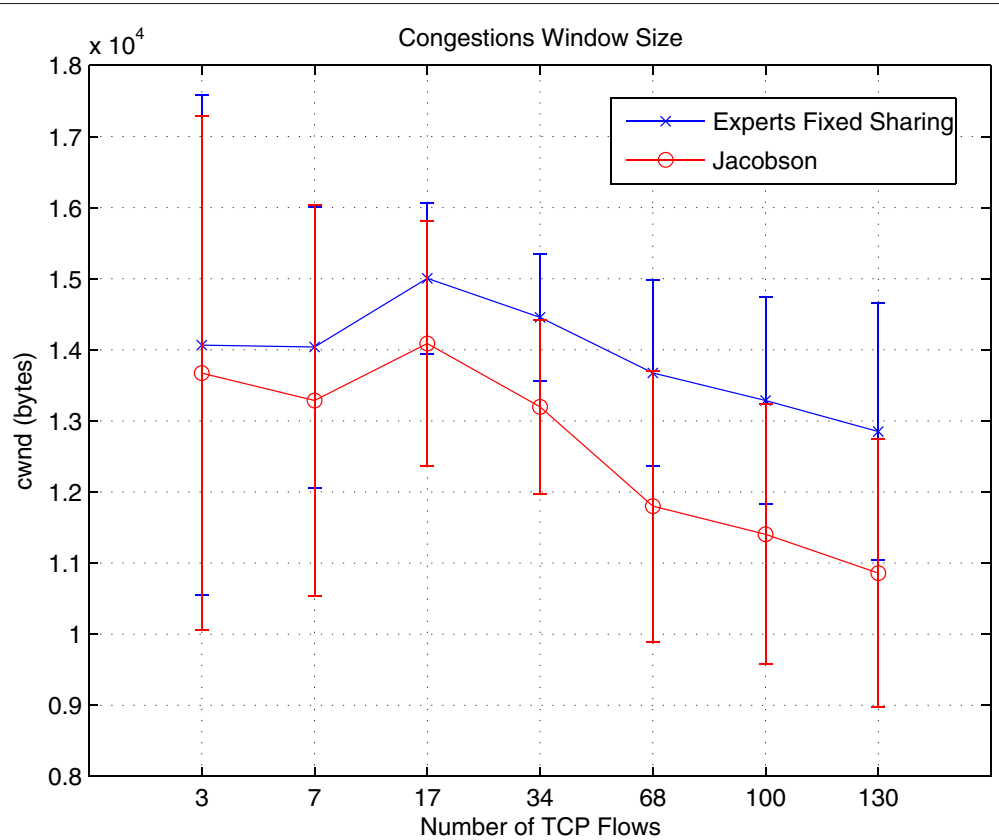

Figure 16 TCP's cwnd over different congestion levels for the stationary scenario. 


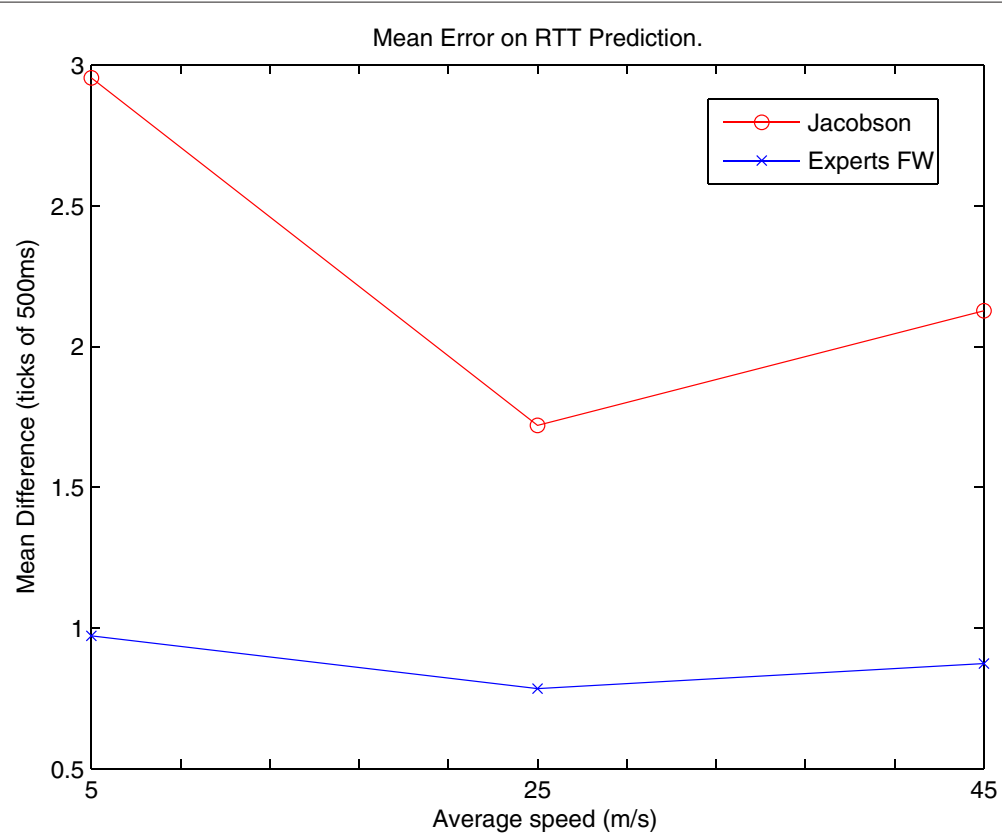

Figure 17 Mean absolute error between predicted and measured RTT for different node speeds in bursty traffic scenario.

in fewer losses and lower routing overhead. For the average speed of $25 \mathrm{~m} / \mathrm{s}$, the situation changes and the metrics reflect the worst performance. However, when further increasing the average speed to $45 \mathrm{~m} / \mathrm{s}$, the network metrics start to improve again. This behavior is consistent with the results presented in [21], which shows that, when topology changes happen at packet delivery time scales, network capacity can improve when nodes are mobile rather than stationary.

\subsection{Scenario V - wired network}

Here, we simulate an eight-node wired network whose topology can be seen in Figure 21. In this scenario, we vary the traffic load by using 30,70 , and 100 concurrent

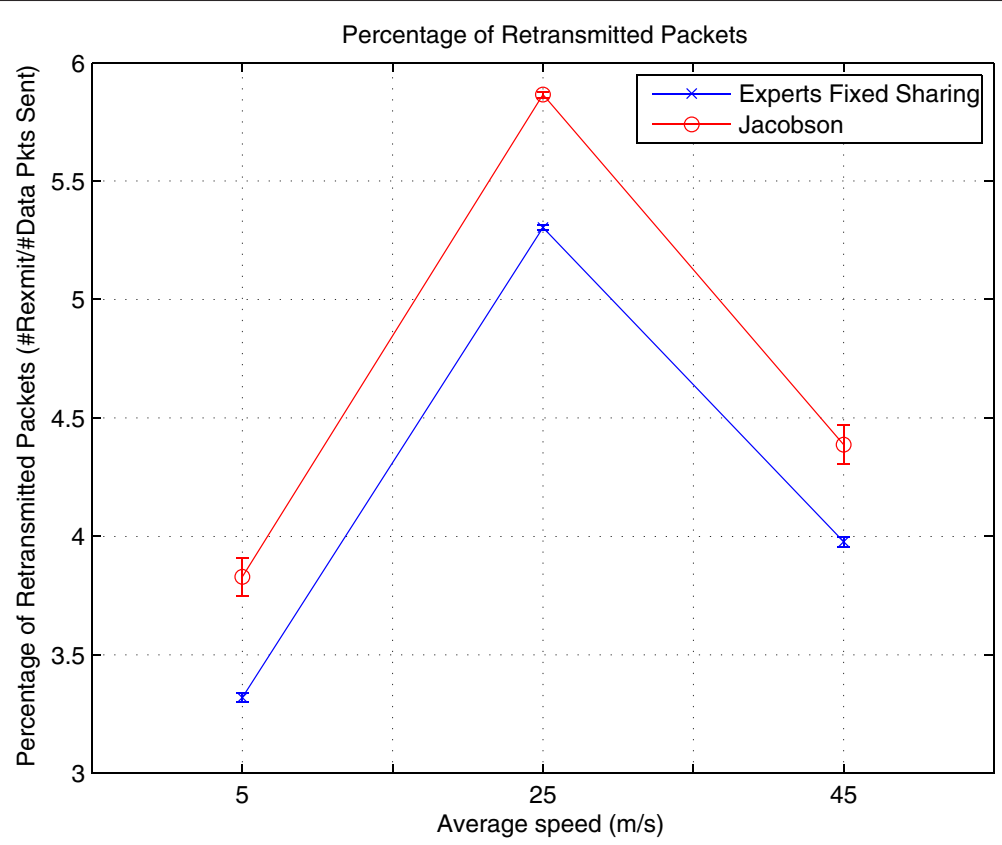

Figure 18 Percentage of retransmitted packets for different node mean speeds in the bursty traffic scenario. 


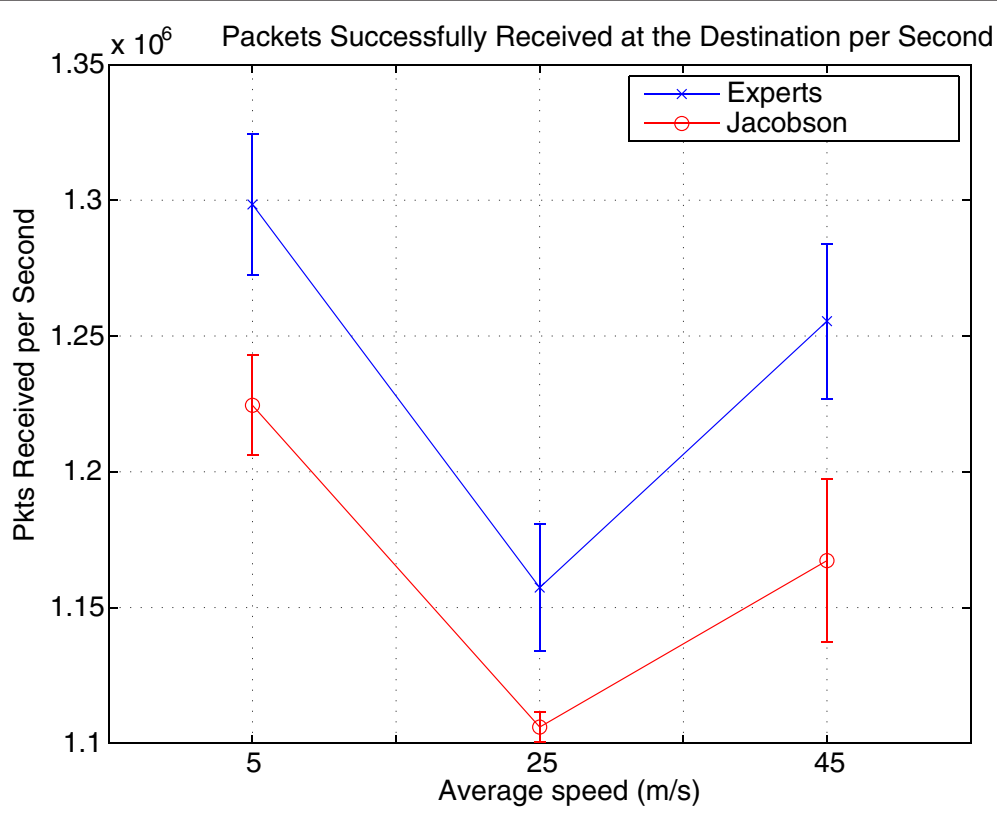

Figure 19 Goodput for different node mean speeds in the bursty traffic scenario.

flows. Flows were evenly distributed among nodes but start at random times throughout the 90-min duration of the simulation. The size of a flow is uniformly distributed between 1,000 and 10,0000 packets.

As shown in Figure 22, the mean prediction error for Jacobson's algorithm is almost four times higher than when using the experts framework. The benefits of the more accurate RTT estimates yielded by our approach is illustrated in Figures 23,24,25 which show lower number of retransmitted packets, higher delivery ratio, and higher goodput as the number of TCP flows increases.

\section{Linux implementation and experiments}

In this section, we present our implementation of the fixed-share experts algorithm for the Linux kernel and report on the experiments we conducted and their results.

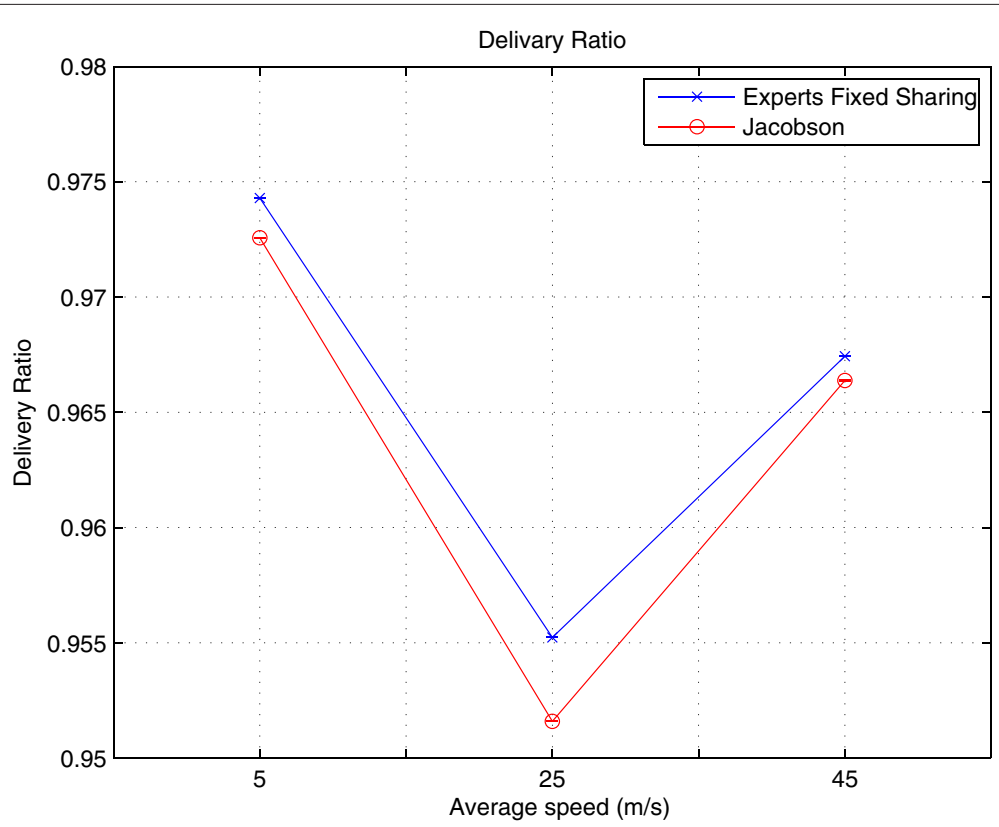

Figure 20 Packet delivery ratio for different node mean speeds in the bursty traffic scenario. 


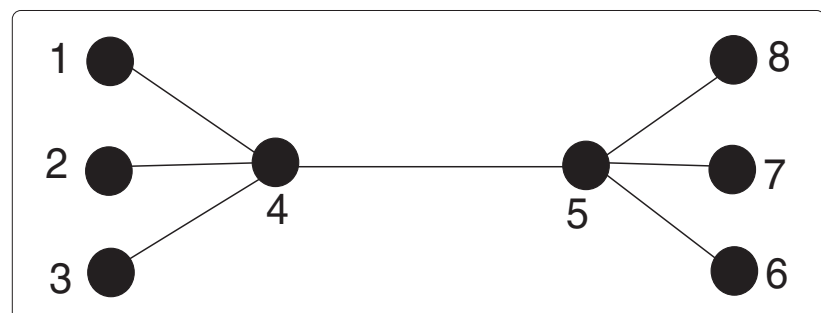

Figure 21 Simulated network topology for the wired scenario.

\subsection{Fixed-point arithmetic}

The simulation results reported in Section 5 refer to the implementation of the fixed-share experts algorithm (as described in Section 3) as implemented on the QualNet network simulator. This implementation uses real numbers. Thus, a straightforward Linux implementation would use floating point arithmetic [22] along with floating point functions of the gcc compiler's libc library [23]. Unfortunately, while floating point numbers support both a wide range of values and high precision, the Linux operating system lacks support for floating point manipulation in the kernel ${ }^{\mathrm{d}}$.

An alternative is to use fixed point arithmetic in which the location of the radix point within a string of digits is predetermined [24]. In our implementation, we define a fixed point arithmetic type with a 16-bit integral part and a 16-bit fractional part as shown below.

$$
s b_{31} \cdots b_{16} \cdot b_{15} \cdots b_{0}
$$

In our Linux implementation we used a sign magnitude representation in which a 33rd bit records the sign (an alternate implementation of the data type would reduce the integer part to 15 bits so that the resulting type would fit entirely within a single 32-bit processor register.)

Our fixed point numeric type has the following characteristics: range $=-65535.99998$ to +65535.99998 and precision $=0.000015$. We consider these characteristics adequate for the range of numeric values expected.

\subsection{Linux implementation}

We implemented both the fixed share experts algorithm and the Eifel algorithm [4] in the Linux kernel version 2.6.28.3. We modified the function tcp_rtt_estimator() to return the output of the RTT as evaluated by either of the RTT prediction algorithms. Our implementation of the Eifel algorithm, to the best of our knowledge, is faithful to the algorithm described in [4] for predicting the RTT and setting the RTO.

Our Linux implementation of the fixed share experts algorithm differs from our QualNet implementation of the algorithm in two areas. First, the implementation scales RTT measurements. A measured RTT of 1 tick in the simulator means $500 \mathrm{~ms}$, while a measured RTT of 1 tick in the Linux implementation means $4 \mathrm{~ms}$. Consequently, our Linux implementation scales RTT measurements from the operating system by $\frac{1}{125}$ before passing them to the fixed share experts algorithm, and it scales RTT predictions from the experts algorithm by 125 before returning

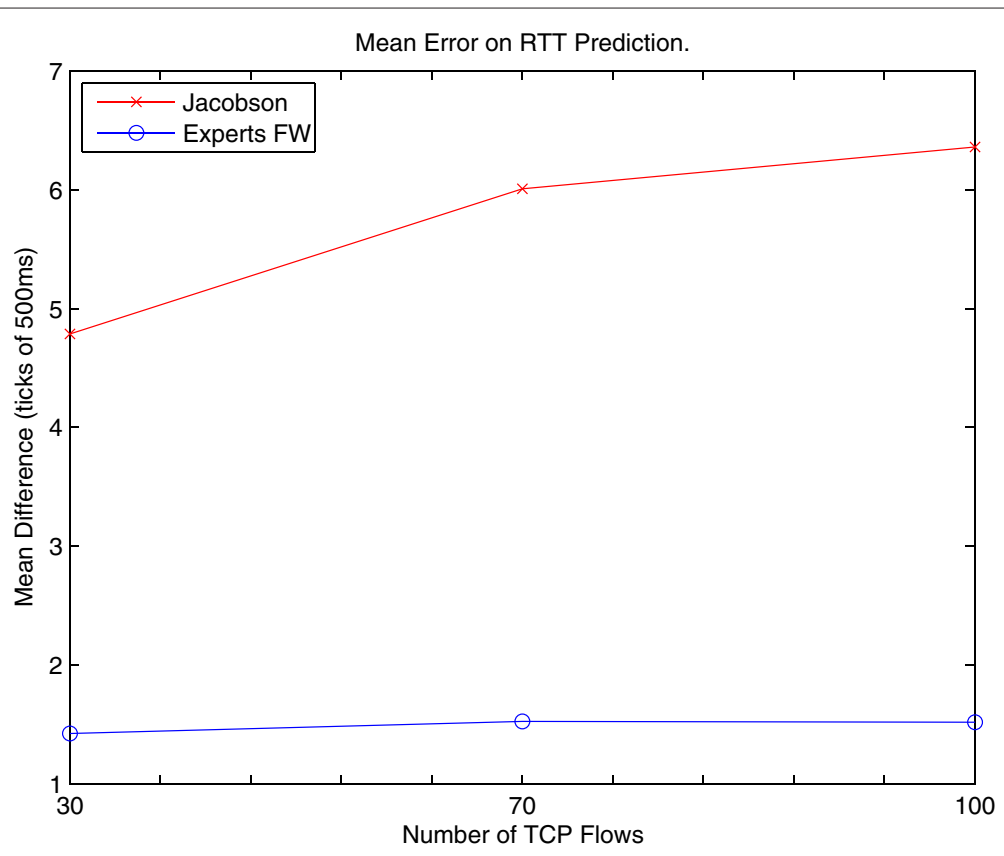

Figure 22 Mean absolute error between the predictions and the measured RTT for the wired scenario. 


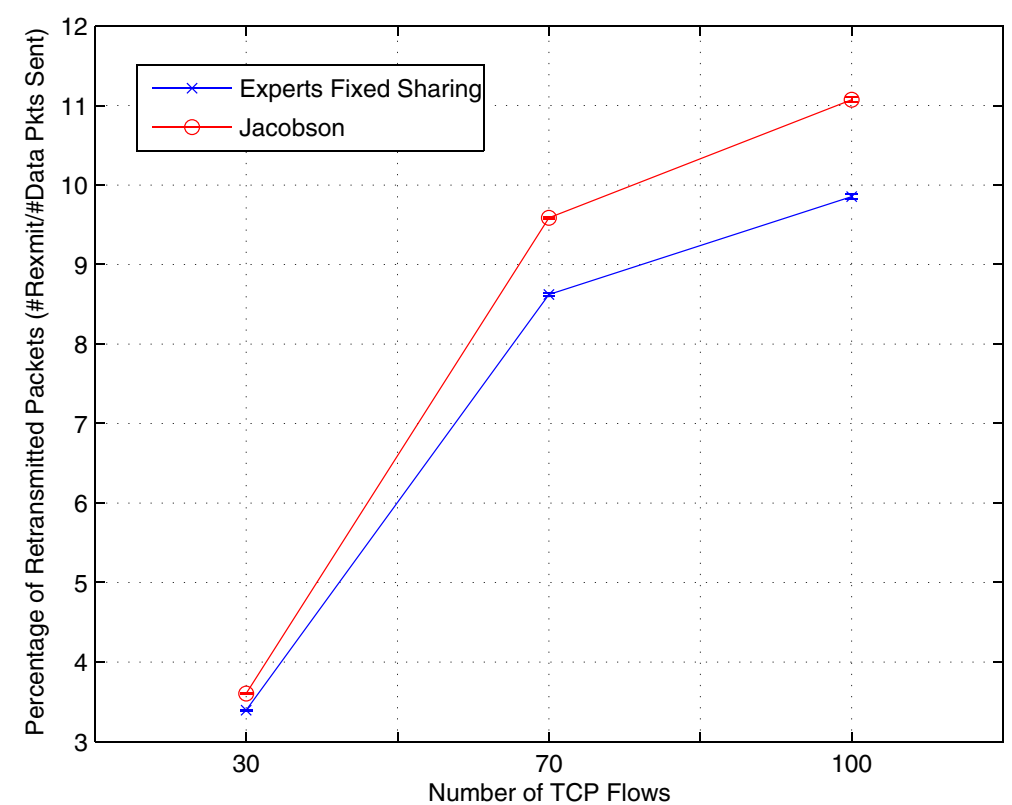

Figure 23 Number of retransmitted packets in relation to the total number of packets transmitted for the wired scenario.

them to the operating system. Such scaling prevents the implemented algorithm from misinterpreting the greater precision of the Linux RTT measurements as larger prediction errors.

The second difference in the Linux implementation of algorithm is inspired by the algorithm's response to large and abrupt reductions in the measured RTTs. Large RTT reductions cause the weights of formerly correct experts to experience greater losses in extreme cases immediately underflowing to 0 . In these cases, if the weights of the newly correct experts already have decayed to zero, then all experts' weights will be zero simultaneously, and the machine learning algorithm will be unable to make a prediction. Normally, the fixed-sharing feature of the experts algorithm helps increase the weights of newly correct experts, but sharing cannot compensate for this

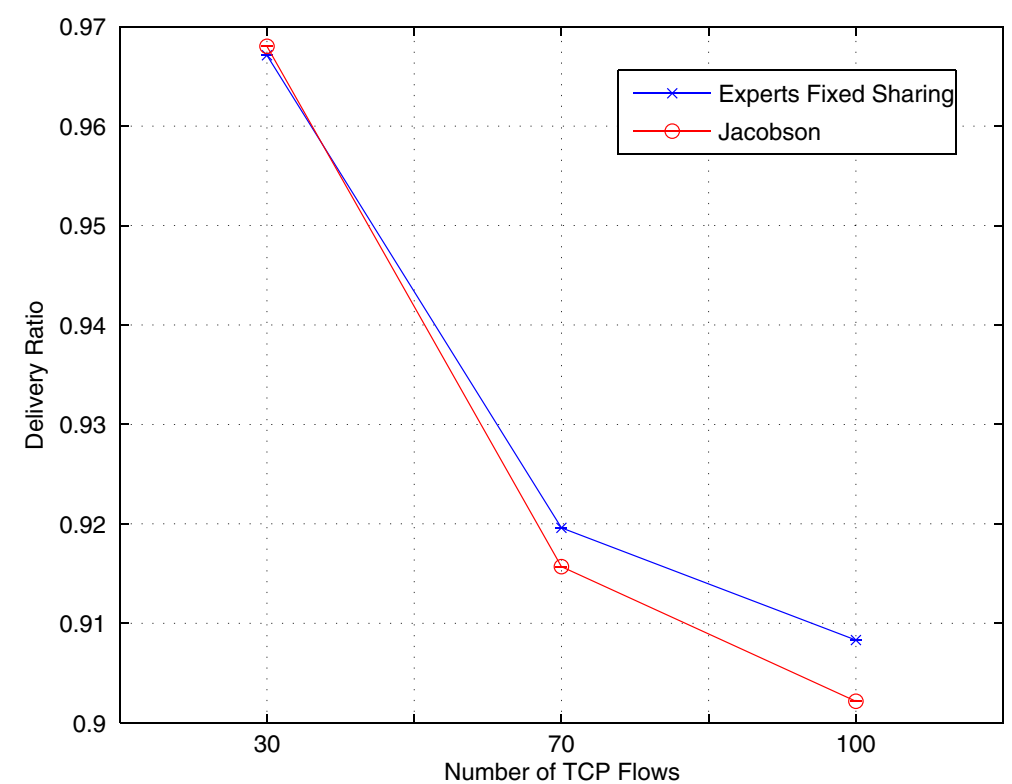

Figure 24 Packet delivery ratio for the wired scenario. 


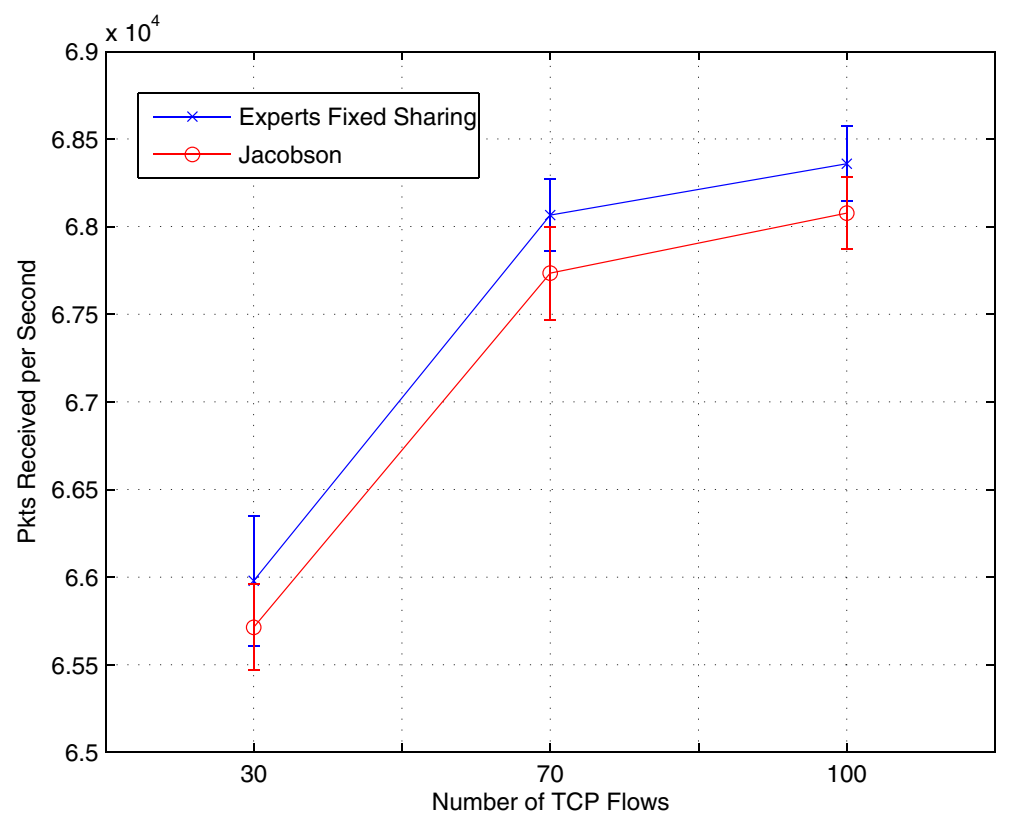

Figure 25 Total number of packets delivered for the wired scenario.

situation since sharing a total weight of zero among the experts has no effect on the experts' individual weights. To compensate for this occasional situation, we modified the algorithm in the Linux implementation to detect the case and to reinitialize the experts' weights with values from a uniform distribution whose mean matches the most recently measured RTT. This change to the algorithm does not affect the simulation results because the simulated RTT changes were sufficient to cause all experts' weights to go to zero.

\subsection{Experimental results}

We acquired data from live file transfer runs using our modified TCP kernel modules that implement the Eifel and the experts algorithms. Data collection happened over 30 file transfers of a 16-MB file. To help filter out the effects of gradual network changes, we interleaved the transfers controlled by our experts approach, the Eifel retransmission timer, and Jacobson's algorithm. In total, there were 10 runs of each algorithm for each of the three conceived scenarios.

The live experiments used a different set of scenarios than the simulations. In Scenario 1, the source of the file transfer was a Linux machine containing the modified modules for the experts and Eifel algorithms and the original Kernel code and TCP timer. This machine was connected to the wired campus network at the University of California, Santa Cruz. The destination was another Linux machine connected to the Internet, physically located in the state of Utah in the USA. Scenario 2 was similar to Scenario 1, except that the source was now connected wirelessly to a 802.11 access point, which was connected to the Internet through the UCSC campus network. Scenario 3 was a full wireless scenario, where both source and destination were connected to the same 802.11 access point. All the measurements were collected at the source of the file transfer.

Figure 26 shows around 200 prediction trials of one of the file transfers. It is possible to notice how much faster the machine learning algorithm can respond to sudden changes in the RTT value and how much closer it can follow the real measurements.

Tables 2, 3, 4 summarize results from live experiments for the three scenarios studied. These tables shows an improvement on the RTT prediction from $40 \%$ in Scenario 1 up to $51 \%$ in Scenario 3 when comparing the accuracy between the experts algorithm and the standard TCP predictor. This difference is even higher when comparing to Eifel. The other performance metrics average number of retransmissions and cwnd - also improved considerably when applying our machine learning approach. On the other hand, Eifel has the advantage of not requiring any parameters to be set since gains are computed 'on-the-fly'. In the case of Jacobson's algorithm, even though a couple of parameters have to be set in advance, it is a much simpler and easier to implement algorithm. However, trading-off complexity to achieve significantly higher performance is consistent with the steady increase of processing and storage capabilities available in computing and communication devices. Thus, given the superior performance illustrated by our results, we can conclude that our approach yields a good 


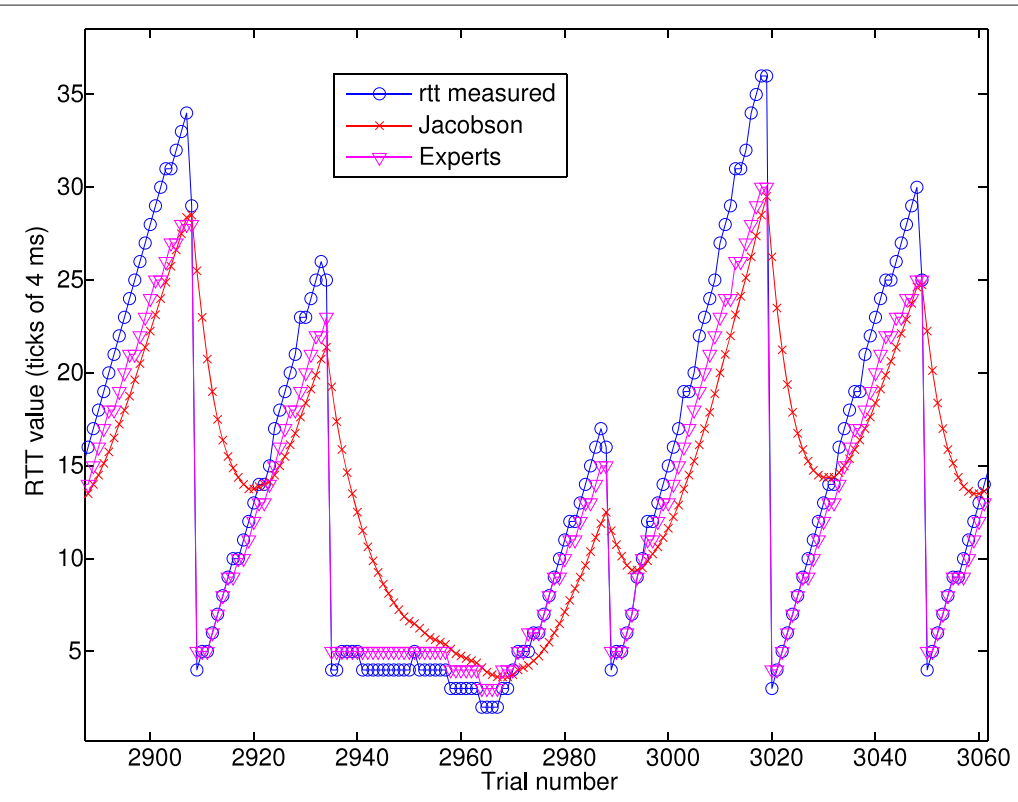

Figure 26 RTT measurements on the Linux kernel and predictions made by Jacobson's and the proposed experts algorithms.

trade-off between higher complexity and performance improvement.

\subsubsection{RTO computation}

In the preliminary experiments, we notice a considerable improvement on the RTT predictions as expected and as seen previously in the simulated results. However, curiously, we experienced a higher number of retransmissions and a lower cwnd. The reason for that was the fact that, when computing the RTTVAR in Equation 2, the difference between the estimation and the RTT sample (|SampleRTT EstimatedRT|) is used. With a better predictor, this difference is much smaller on the average, which makes the timer much more aggressive. During the simulations, it was not a problem because the RTT values fluctuate over a range that was 125 times smaller, as mentioned in the previous section. In order to fix this problem, we made a simple change in the way the RTTVAR is computed. Before,

Table 2 Prediction error, cwnd, and number of retransmissions averaged over 10 runs of the same experiment

\begin{tabular}{lccc}
\hline \multirow{2}{*}{ Metric } & \multicolumn{3}{c}{ Scenario1 } \\
\cline { 2 - 4 } & Eifel & Jacobson & Experts \\
\hline Error & $11.21_{(1.02)}$ & $8.19(0.97)$ & $5.10(0.61)$ \\
cwnd & $61.55_{(10.42)}$ & $69.82_{(6.32)}$ & $74.87_{(9.73)}$ \\
rexmits & $26.40_{(13.83)}$ & $31.12_{(17.72)}$ & $13.02(8.24)$ \\
\hline
\end{tabular}

Prediction error (in ticks), cwnd (in packets), and number of retransmissions averaged over 10 runs of the same experiment, computed for Scenario 1. Values between parenthesis depict the standard deviation $\sigma$ over the 10 runs. Italicized values indicate the smalest error, largest cwnd, and lowest number of retrasmission. when using Jacobson's algorithm, it made sense to use the difference between sample and estimation since the estimation was a smoothed tracking of the RTT sample, and the RTTVAR would indicate how much variation around that smoothed value the RTT measurements experience. Now with the new predictor tracking the RTT measurements much faster, we used the difference between the current and last RTT sample to compute the RTTVAR, as indicated in Equation 4.

$$
\mathrm{RTTVAR}=(1-\beta) \cdot \mathrm{RTTVAR}+\beta \cdot \mid \text { SampleRTT } \mathrm{t}-\text { SampleRTT }_{\mathrm{t}-1} \mid
$$

\section{Conclusions}

In the present work, we proposed a novel approach to end-to-end RTT estimation using a machine learning technique known as the fixed-share experts framework.

Table 3 Prediction error, cwnd, and number of retransmissions averaged over 10 runs of the same experiment for Scenario 2

\begin{tabular}{lccc}
\hline \multirow{2}{*}{ Metric } & \multicolumn{3}{c}{ Scenario2 } \\
\cline { 2 - 4 } Error & Eifel & Jacobson & Experts \\
cwnd & $114.52_{(9.15)}$ & $74.11_{(6.23)}$ & $67.64_{(4.64)}$ \\
rexmits & $55.38_{(8.34)}$ & $66.71_{(5.35)}$ & $74.89_{(7.03)}$ \\
\hline
\end{tabular}

Prediction error (in ticks), cwnd (in packets), and number of retransmissions averaged over 10 runs of the same experiment, computed for Scenario 2. Values between parenthesis depict the standard deviation $\sigma$ over the 10 runs. Italicized values indicate the smalest error, largest cwnd, and lowest number of retrasmission. 
Table 4 Prediction error, cwnd, and number of retransmissions averaged over 10 runs of the same experiment for Scenario 3

\begin{tabular}{lccc}
\hline & \multicolumn{3}{c}{ Scenario3 } \\
\cline { 2 - 4 } Metric & Eifel & Jacobson & Experts \\
\hline Error & $298.24_{(21.41)}$ & $199.23(12.32)$ & $131.65(7.58)$ \\
cwnd & $18.91_{(6.68)}$ & $31.08(7.38)$ & $38.11(5.91)$ \\
rexmits & $204.62_{(32.43)}$ & $363.21_{(39.86)}$ & $159.61(24.18)$ \\
\hline
\end{tabular}

Prediction error (in ticks), cwnd (in packets), and number of retransmissions averaged over 10 runs of the same experiment, computed for Scenario 3. Values between parenthesis depict the standard deviation $\sigma$ over the 10 runs. Italicized values indicate the smalest error, largest cwnd and lowest number of retrasmission.

We employ our approach as an alternative to TCP's RTT estimator and show that it yields higher accuracy in predicting the RTT than the standard algorithm used in most TCP implementations. The proposed machine learning algorithm is able to adapt very quickly to changes in the RTT. Our simulation results show a considerable reduction in the number of retransmitted packets, while increasing goodput, particularly in more heavily congested scenarios. We corroborate our results by running 'live' experiments on a Linux implementation of our algorithm. These experiments confirm the higher accuracy of the machine learning approach with more than $40 \%$ improvement, not only over the standard TCP predictor but also when comparing to another well know solution, the Eifel retransmission timer [4]. Nevertheless, work is still needed in the case of this particular application in order to learn how to take better advantage of the improved estimations and change the way we set the RTO timer.

Moreover, the task of determining the appropriate loss function for RTT prediction in the case of setting retransmission timers is not trivial. Further work to understand the cost of making wrong decisions regarding the RTT prediction problem, under the context of TCP, is needed. Finally, we believe our work opens the possibility of applying on-line learning algorithms to predict other important network variables.

\section{Endnotes}

${ }^{a}$ In this paper, we use the terms experts framework, experts FW, experts fixed sharing, fixed-share experts, or simply the experts algorithm, interchangeably, referring to the proposed machine learning algorithm.

${ }^{\mathrm{b}}$ These values were used in our simulations; however, on real implementations, they can vary. That was the case for the TCP implementation on the Linux distribution used in our experiments, and we comment on that in Section 6.

${ }^{\mathrm{c}}$ The solution proposed by Eifel for this problem (not the algorithm itself) made it to recent TCP kernel implementations and were used in our experiments reported in Section 6.

${ }^{\mathrm{d}}$ Within the Linux kernel, one can surround in-line floating point code with the Linux macros kernel_fpu_begin and kernel_fpu_end, but the code must avoid function calls and must avoid using any routines of the libc library.

\section{Competing interests}

The authors declare that they have no competing interests.

\section{Acknowledgements}

Financial support was granted by the CAPES Foundation Ministry of Education of Brazil, Caixa Postal 250, Brasilia - DF 70040-020 Brazil. This work was also partially supported by NSF grant CCF-091694 and a US Army-ARO MURI grant.

\section{Author details}

${ }^{1}$ Department of Computer Engineering, Baskin School of Engineering University of California, Santa Cruz, CA 95064, USA. ${ }^{2}$ Department of Computer Science, Columbia University, New York, NY 10027, USA. ${ }^{3}$ Department of Computer Science, Loyola University Maryland, Baltimore, MD 21210, USA.

Received: 5 June 2013 Accepted: 12 March 2014

Published: 26 March 2014

\section{References}

1. Akamai Technologies, Inc. http://www.akamai.com. Last accessed, Dec. 1st 2013

2. M Herbster, MK Warmuth, Tracking the best expert. Mach. Learn. 32(2), 151-178 (1998)

3. V Jacobson, Congestion avoidance and control. SIGCOMM Comput. Commun. Rev. 25, 157-187 (1995)

4. R Ludwig, K Sklower, The Eifel retransmission timer. SIGCOMM Comput. Commun. Rev. 30(3), 17-27 (2000)

5. P Karn, C Partridge, Improving round-trip time estimates in reliable transport protocols. ACM Trans. Comput. Syst. 9, 2-7 (2001)

6. M Allman, V Paxson, On estimating end-to-end network path properties, in SIGCOMM '99: Proceedings of the Conference on Applications, Technologies, Architectures, and Protocols for Computer Communication (ACM New York, 1999), pp. 263-274

7. W Lou, C Huang, Adaptive timer-based TCP control algorithm for wireless system, in Wireless Networks, Communications and Mobile Computing, IEEE International Conference on, Volume 2 (Maui, HI, USA, 2005), pp. 935-939

8. L Ma, G Arce, K Barner, TCP retransmission timeout algorithm using weighted medians. Signal Process. Lett. IEEE. 11(6), 569-572 (2004)

9. K Leung, T Klein, T Mooney, T Haner, Methods to improve TCP throughput in wireless networks with high delay variability [3G network example], in Vehicular Technology Conference, 2004. VTC2004-Fall. 2004 IEEE 60th, Volume 4 (Los Angeles, CA, USA, 2004), pp. 3015-3019

10. DP Helmbold, DDE Long, B Sherrod, A dynamic disk spin-down technique for mobile computing, in MobiCom '96: Proceedings of the 2nd Annual International Conference on Mobile Computing and Networking (ACM New York, 1996), pp. 130-142

11. M Haeri, A Rad, TCP retransmission timer adjustment mechanism using model-based RTT predictor, in Control Conference, 5th IEEE Asian, Volume 1 (Melbourne, Australia, 2004), pp. 686-693

12. D Ngwenya, G Hancke, Estimation of SRTT using techniques from the practice of SPC and change detection algorithms, in AFRICON, 2004. IEEE 7th AFRICON Conference in Africa, Volume 1 (Gaborone, Botswana, 2004), pp. 397-402

13. AF Atlasis, NH Loukas, AV Vasilakos, The use of learning algorithms in ATM networks call admission control problem: a methodology. Comput. Netw. 34(3), 341-353 (2000)

14. A Vasilakos, M Saltouros, AF Atlassis, W Pedrycz, Optimizing QoS routing in hierarchical ATM networks using computational intelligence techniques. Syst. Man Cybernet. Part C: Appl. Rev. IEEE Trans. 33(3), 297-312 (2003)

15. M Anastasopoulos, D Petraki, R Kannan, A Vasilakos, TCP throughput adaptation in WiMax networks using replicator dynamics. Syst. Man Cybernet. Part B: Cybernet. IEEE Trans. 40(3), 647-655 (2010) 
16. M Mirza, M Sommers, P Barford, X Zhu, A machine learning approach to TCP throughput prediction. SIGMETRICS Perform. Eval. Rev. 35, 97-108 (2007)

17. O Bousquet, MK Warmuth, Tracking a small set of experts by mixing past posteriors. J. Mach. Learn. Res. 3, 363-396 (2003)

18. QualNet. http://www.scalable-networks.com. 6100 Center Drive, Suite 1250, Los Angeles, CA 90045. Last accessed, Dec. 1st 2013

19. C Perkins, E Royer, Ad-hoc On-Demand Distance Vector Routing, in Proceedings of the 2nd IEEE Workshop on Mobile Computing Systems and Applications (New Orleans, LA, USA, 1997), pp. 90-100

20. IW Group, Wireless LAN Medium Access Control (MAC) and Physical Layer (PHY) Specification. IEEE Std. 802.11, 345 E. 47th St, New York, NY 10017 (IEEE Computer Society, USA, 1997)

21. M Grossglauser, D Tse, Mobility increases the capacity of ad hoc wireless networks. Netw. IEEE/ACM Trans. 10(4), 477-486 (2002)

22. IEEE Computer Society Standards Committee Working group of the Microprocessor Standards Subcommittee, American National Standards Institute, IEEE Standard for Binary Floating-point Arithmetic. (IEEE Computer Society, ANSI/IEEE Std 754-1985., 345 E. 47th St, New York, NY, 10017, USA, 1985)

23. The GNU C Library. http://www.gnu.org/software/libc/manual. (51 Franklin Street, Fifth Floor, Boston, MA 02111, USA 2009). Last accessed, Dec. 1st 2013

24. AR Omondi, Computer Arithmetic Systems: Algorithms, Architecture, and Implementation. (Prentice Hall International (UK) Limited, 1994)

Cite this article as: Nunes et al:: A machine learning framework for TCP round-trip time estimation. EURASIP Journal on Wireless Communications and Networking 2014 2014:47.

\section{Submit your manuscript to a SpringerOpen ${ }^{\mathcal{O}}$ journal and benefit from:}

- Convenient online submission

- Rigorous peer review

- Immediate publication on acceptance

- Open access: articles freely available online

- High visibility within the field

- Retaining the copyright to your article

Submit your next manuscript at springeropen.com 